\title{
Lateralized task shift effects in Broca's and Wernicke's regions and in visual word form area are selective for conceptual content and reflect trial history
}

\author{
Mikkel Wallentin ${ }^{\mathrm{a}, \mathrm{b}, *}$, Jákup Ludvík Dahl Michaelsen ${ }^{\mathrm{b}}$, Ian Rynne ${ }^{\mathrm{b}}$, Rasmus Høll Nielsen ${ }^{\text {b }}$ \\ a Center of Functionally Integrative Neuroscience, Aarhus University Hospital, Building 10-G-5, Nørrebrogade, 8000 Aarhus C, Denmark

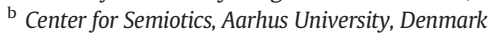

\section{A R T I C L E I N F O}

Article history:

Accepted 8 July 2014

Available online 15 July 2014

\begin{abstract}
A B S T R A C T
We investigated whether lateralized BOLD-fMRI activations in Broca's region, Wernicke's region and visual word form area (VWFA) reflect task shift costs and to which extent these effects are specific to language related task shifts. We employed a linguistic one-back memory paradigm where participants $(n=58)$ on each trial responded to whether a given word was the same as the previous word. In concordance with previous findings we found that conceptual shifts (CS), i.e. new words, elicited a strongly left-lateralized response in all three regions compared to repeat words. Words were sometimes presented through the visual modality (read) and sometimes through the auditory modality (spoken). This enabled the study of perceptual modality shifts (PS) relative to trials that stayed in the same modality as the previous trials. Again, we found a strongly left-lateralized effect in all regions. This was independent of whether the word was a CS or not, suggesting that linguistic translation across modalities taxes the same system as CS. Response shifts (RS), on the other hand, when shifting from one response (e.g. reporting a repeat word) to another (e.g. reporting a new word) did not yield an observable left lateralized response in any of the regions, suggesting that the lateralized task shift cost effects in these regions are not shared by all types of task shifts. Lateralization for individual tasks was found to be correlated across brain regions, but not across tasks, suggesting that lateralization may not be a unitary phenomenon, but vary across participants according to task demands. Both response time and lateralization were found to reflect the demands not only of the current trial but also of the previous trial, illustrating the context dependency of even simple cognitive tasks.
\end{abstract}

(c) 2014 Elsevier Inc. All rights reserved.

\section{Introduction}

Broca's region

Broca's region (Amunts and Zilles, 2012; Broca, 1861), defined as Brodmann areas 44 and 45 of the left inferior frontal gyrus (LIFG) is known to play a key role in speech production as well as in the processing of language input (e.g. see Kristensen and Wallentin, in press for a review). The exact function (or functions) of Broca's region, however, remains disputed (Grodzinsky and Santi, 2008; Novick et al., 2010). Recently, it has been suggested that activation in this area reflects general linguistic processing costs (Kristensen and Wallentin, in press). In support of this are findings that syntactic manipulations yield increased Broca's region activation (Ben-Shachar et al., 2004; Christensen and Wallentin, 2011; Kristensen et al., 2013; Tettamanti et al., 2009) and findings showing that Broca's region responds to

\footnotetext{
* Corresponding author at: Center of Functionally Integrative Neuroscience, Aarhus University Hospital, Building 10-G-5, Nørrebrogade, 8000 Aarhus C, Denmark.

E-mail address: mikkel@cfin.dk (M. Wallentin).
}

unpredicted word order, rather than syntactic manipulations per se, as defined by working memory demands (Fiebach et al., 2005), cloze probability (Obleser and Kotz, 2010) or context (Kristensen et al., in press).

Broca's region activation can also be observed in the absence of word order manipulations. Single word studies have demonstrated that Broca's region activity is correlated with working memory demand (Cohen et al., 1997), and displays an inverse correlation with word frequency (Fiebach et al., 2002), word generation practice (Raichle et al., 1994) and priming (Thompson-Schill et al., 1999). Pseudowords, on the other hand, generate greater activation than real words (Fiebach et al., 2002), again suggesting that unexpected words or words that are difficult to understand yield greater activation. Along the same lines, Broca's region has been found to increase activation to degraded visual word features (visual word form) as well as to decreased plausibility based on world knowledge (van de Meerendonk et al., 2013). More generally, it has been found that Broca's region activation is positively correlated with response time during linguistic processing (Christensen and Wallentin, 2011; Kristensen and Wallentin, in press; Orfanidou et al., 2006; Wallentin et al., 2006). 
The question remains, however, at what level of abstraction this effect occurs (Fedorenko et al., 2013). Is this a general linguistic phenomenon as the diverse findings seem to suggest, and if so, is it limited to language or can it be observed with tasks that are non-linguistic as well? Recent proposals suggest that Broca's region serves a more domain general purpose during cognitive control (Fedorenko et al., 2013; Novick et al., 2010).

\section{Wernicke's region}

Wernicke's region, usually defined as the posterior part of the superior temporal cortex behind the primary auditory cortex (Geschwind, 1970; Perry and Lupyan, 2014; Price, 2000), is traditionally thought to be involved in the recognition of patterns of spoken language (Geschwind, 1970; Wernicke, 1874). However, discussions similar to those for Broca's region have been led on the exact function, location and even existence of Wernicke's region (e.g. Binder et al., 1996; Bogen and Bogen, 1976; Dronkers et al., 2004; Turken and Dronkers, 2011; Wise et al., 2001). Recent studies have suggested that Wernicke's region plays a role in the mapping between visual and linguistic representations of categories (Perry and Lupyan, 2014) and indeed perhaps the most compelling argument in favor of the posterior temporal cortex being involved in linguistic processing is the location right between auditory and visual cortices (Geschwind, 1970), making it intuitively appealing to believe that it serves a function as an associative region for input that link the two perceptual modalities.

\section{Visual word form area}

In the posterior part of the inferior temporal gyrus lies a region that has repeatedly been found to be associated with reading words, so much that it has been named the visual word form area - VWFA (Dehaene and Cohen, 2011; Dehaene et al., 2002). VWFA has been found to be functionally specific to words relative to objects (Szwed et al., 2011) and relative to mirror images of words (Dehaene et al., 2010a). It has also been found to be sensitive to bigram frequency, i.e. the statistics of letter pairs in a particular language (Vinckier et al., 2007). However, controversies also exist about this alleged specificity (Price and Devlin, 2003) with some also finding context dependency of the VWFAs response pattern (Starrfelt and Gerlach, 2007) Additionally, VWFA has been found to be involved in the processing of both spoken words and pseudo-words (Orfanidou et al., 2006). Although more ventrally placed than Wernicke's region, the VWFA may thus also be a region relevant for the interface between visual and auditory aspects of word forms.

\section{Lateralization of Broca's and Wernicke's regions and VWFA}

The question about the specificity of Broca's region, Wernicke's region and VWFA activity corresponds to a similar discussion about lateralization in cognitive tasks and language in particular. Language function has been found to be strongly left lateralized when studied through deficits elicited either by brain injury (Dronkers et al., 2004; Pedersen et al., 1995; Pedersen et al., 2004) or by experimentally induced temporary deficits (Rasmussen and Milner, 1977; Woermann et al., 2003). Wernicke's region has long been known to be structurally asymmetric (Gannon et al., 1998; Geschwind and Levitsky, 1968). Activation in the VWFA has, similar to Broca's region, been found to be left-lateralized (Szwed et al., 2011). But the functional effects observed for all regions using functional imaging have often been much less lateralized than the patient studies would predict (e.g. Binder et al., 2000; Brown et al., 2006; Cardillo et al., 2012; Ferstl et al., 2008; Petersen et al., 2013; Vigneau et al., 2006, 2011). One reason for this discrepancy between patient and neuroimaging findings might be that lateralization indices from fMRI studies often are not based on close contrasts between linguistic inputs that differ in terms of processing cost. Indeed, when contrasting linguistic tasks to baseline, a bilateral pattern is often observed (Ferstl et al., 2008). Another reason might be that fMRI based lateralization indices are most often derived from a count of activated voxels in each hemisphere (Bethmann et al., 2007; Binder et al., 2000; Cai et al., 2010; Clements et al., 2006; Deppe et al., 2000; Lehéricy et al., 2000; Pujol et al., 1999; Springer et al., 1999; Szaflarski et al., 2002; Szaflarski et al., 2012; Woermann et al., 2003), a method that is very sensitive to the chosen significance threshold (Jones et al., 2011; Seghier, 2008; Suarez et al., 2009). But the divergent findings also point towards the possibility that lateralization may not be uniform across tasks and brain regions. One example is music. Lateralization for music has been found to depend on musical competence, both when studied using fMRI and MEG (Vuust et al., 2005, 2006). Similarly, it has been found that activation and lateralization in the VWFA change as a function of increased literacy (Dehaene et al., 2010b). But literacy is independent of musical competences and the lateralization changes relevant for the two task types therefore must be hypothesized to happen independently. This can only be the case if there is more than one type of lateralization. This calls for an investigation of whether lateralization is linked to individual brain regions (region specific) or whether lateralization is rather linked to the type of task at hand (task specific) or both.

\section{Hypotheses}

In the current experiment we investigated to which extent Broca's region (BAs $44 \& 45$ ), Wernicke's region (posterior part of the superior temporal gyrus) and VWFA activity are specific to different types of processing costs elicited by more or less language related task shifts. To this end, we employed a single word one-back memory paradigm where participants responded to whether a given word was the same as the previous word or not (i.e. a conceptual shift), regardless of the perceptual mode of presentation. Stimuli were equally often presented visually or through headphones. Participants were requested to respond as quickly and accurately as possible using a two-finger response box.

We hypothesized that a conceptual shift contrast (CS: new vs repeat words), in accordance with previous findings (Cohen et al., 1997), would elicit increased BOLD activity in Broca's region and that this effect would be strongly left-lateralized. A one-back task that stays within a single perceptual modality can be solved using perceptual surface features alone. But since trials in the current one-back paradigm shifted between visual and auditory formats, this was not always possible. A perceptual shift (PS: new modality vs same modality) from one modality to another would therefore increase the load on the linguistic system, because it would force the participant to re-encode the words at a higher level than the mere perceptual surface, i.e. by translating the perceptual features into the other perceptual modality or by remembering conceptual content. We hypothesized that this process would also result in an increased lateralized Wernicke's region and/or VWFA region activity (Dehaene and Cohen, 2011; Dehaene et al., 2002). The randomized string of stimuli made it possible for a number of new words or repeat words to appear in succession. These trains of repeated trial types would thus allow the participants to respond with the same finger on a number of trials, but whenever a trial went from being a new word to being a repeat word or vice versa this required the participants to perform a response shift (RS: new response vs same response). We hypothesized that these shifts, similar to the two other types of shifts, would elicit prolonged response times, but that this type of added processing cost would not be linguistic in nature and therefore would not show up as strongly left-lateralized responses in Broca's region, Wernicke's region or VWFA. For quantification of lateralization we employed an analysis based on averaging betaestimates across the entire regions of interest. Lastly, we wanted to investigate the possible relationship between lateralization, task and region. Is lateralization task and/or region-specific? If lateralization is task specific then we would expect lateralization indices across the different regions to be correlated within a given task, whereas if it is region 
specific then we would expect lateralization within a region to be correlated across tasks. Lastly, if lateralization is unitary, we should expect it to be correlated across both tasks and regions.

\section{Material and methods}

Participants

Permission for the study was obtained from the local ethics committee (Region Midtjylland, Denmark) and written informed consent was obtained from each participant. Sixty participants with no known neurological history took part in the experiment (28/32 males/females; median age: 22 years, range: $19-38$ years). All participants reported being right-handed and having Danish as their primary language. Each received a 150 DKK payment for participation. One participant was excluded due to a misunderstanding of the instructions and one due to a technical problem during fMRI data acquisition $(\mathrm{n}=58)$.

\section{Stimuli and procedure}

Stimuli consisted of 250 concrete nouns, either displayed on a screen or played through headphones. The words were a subset of a larger collection of words (Sudre et al., 2012) that could be unambiguously translated into Danish. Word meaning spanned a wide area of the semantic space of concrete nouns, but the content is of lesser importance for the present purpose, since all contrasts are based on differences between the same words displayed in different contexts within and across participants. The experiment was a one-back word comparison task with 400 trials consisting of 250 new word trials and 150 repeat word trials. All new words were considered as concept shifts since the word was different from the previous. 200 trials consisted of written words and 200 trials heard. Perceptual shift (PS) trials occurred whenever a trial was presented in a different modality compared to the previous trial, regardless of whether it was a CS trial or not. Participants responded as fast as possible (using right index/middle finger) to whether the current word was the same (index finger response) as the previous or not (middle finger response), regardless of perceptual modality (see Fig. 1). Response shifts (RS) occurred whenever the required response was different from the previous trial, regardless of whether the shift went from new to repeat or from repeat to new and regardless of modality. Order of stimuli and perceptual modality of presentation were randomized within and across participants. All words were recorded in a neutral male voice $(44,100 \mathrm{~Hz}$ sample rate, 16 bit, mono) using the microphone from a laptop computer and Audacity software (http://audacity.sourceforge.net/). The auditory stimuli had a mean duration of $638 \mathrm{~ms}$ (std: $172 \mathrm{~ms}$; range: $255-1068 \mathrm{~ms}$ ). Written words were displayed for $700 \mathrm{~ms}$, and stimulus onset asynchrony was jittered using a skewed distribution (median 2975 ms; range: 2500-5000 ms).

Stimuli were presented and responses obtained using Cogent 2000 (http://www.vislab.ucl.ac.uk/cogent_2000.php), executed in MATLAB. Sounds were presented through a pair of electrodynamic headphones (MR confon, Magdeburg, Germany). These also helped to attenuate scanner noise. During post scan debriefing, all participants reported being able to hear the words through the scanner noise. Visual stimuli were projected onto a screen at the head end of the scanner bed and viewed through a mirror mounted on the head coil.

The whole experiment lasted approximately $20 \mathrm{~min}$. Before entering the scanner, participants were given a trial run of the task with 10 words not used in the experiment in order to get accustomed to the study design and response procedure.

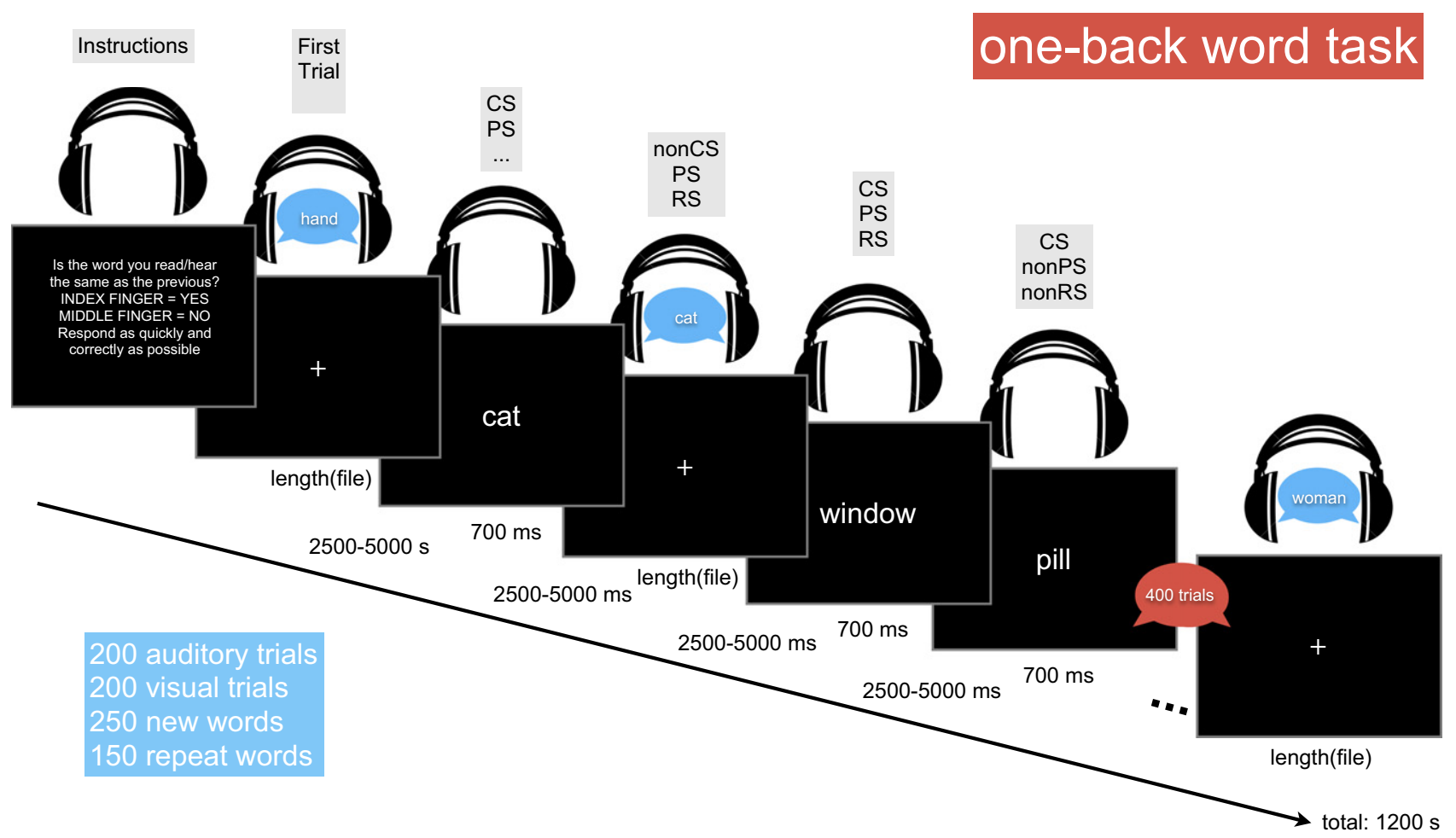

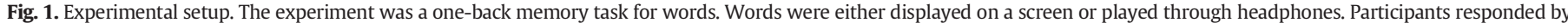

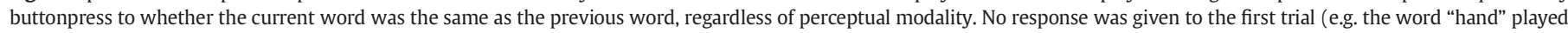

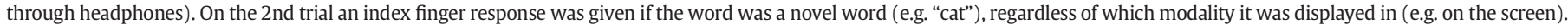

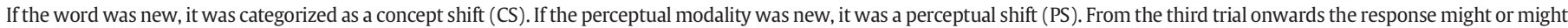

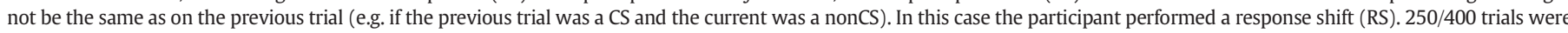

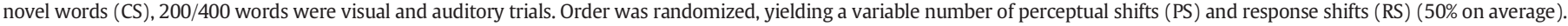




\section{fMRI acquisition}

A 3 T Siemens Magnetom MRI Trio system (Erlangen, Germany) with a 12 channel matrix head coil was used to acquire both T2*-weighted gradient echo, echo-planar images (EPI) with Blood oxygenation level-dependent (BOLD) contrast and $\mathrm{T}_{1}$-weighted structural images. 610 EPI volumes were acquired per participant, not including the first 5 volumes that were discarded to allow for effects of T1 equilibrium. Whole brain coverage was achieved using 40 axial slices (interleaved acquisition) of $3 \mathrm{~mm}$ thickness with an in-plane resolution of $3 \times 3 \mathrm{~mm}$ in a $64 \times 64$ voxel matrix (FOV $192 \mathrm{~mm}$ ). Images were obtained with a TR of $1990 \mathrm{~ms}$, a $27 \mathrm{~ms}$ TE and a $90^{\circ}$ flip angle.

\section{Data analysis}

\section{Pre-processing of fMRI data}

All fMRI image pre-processing and data analyses were performed using Statistical Parametric Mapping software (SPM8; Wellcome Department of Imaging Neuroscience, www.fil.ion.ucl.ac.uk/spm), implemented in MATLAB. Functional images were motion corrected and registered to the first EPI image. The mean of the motioncorrected images was then co-registered to the individual's structural MRI using a 9-parameter affine transformation. The anatomical image was spatially normalized to the standard MNI space using the unified segmentation-normalization algorithm within SPM (Ashburner and Friston, 2005) with the resulting deformation field subsequently applied to the fMRI data. Finally, data were spatially smoothed with an isotropic $8 \mathrm{~mm}$ full width at half maximum (FWHM) Gaussian kernel to account for differences between participants.

\section{Statistical analyses of fMRI data}

Statistical analyses of fMRI data were performed using a two-level general linear model approach (Penny and Holmes, 2007; Worsley and Friston, 1995). In order to control false positives, we thresholded results at $\mathrm{p}<0.05$, family-wise error corrected for multiple comparisons. Main effects were additionally thresholded to only display betaestimates $>1$ or $<-1$ with a cluster size of $>10$ voxels (at the 2 nd contrast level) in order to only report robust effects. In subsequent conjunction analyses we were also concerned with false negatives, so we therefore relaxed the statistical threshold to $\mathrm{p}<0.001$, uncorrected for multiple comparisons, and looked for voxels that would be significant at this level over more than one contrast. Lastly, we performed three regions of interest (ROI) analyses, by averaging beta-estimates across 1) Broca's region and its right hemisphere homologue, 2) Wernicke's region and its right hemisphere homologue and 3) VWFA and its right hemisphere homologue and conducting withinparticipant analyses of variance on the resulting data for each region. The Broca's region ROI was defined using the Wake Forest University Pickatlas (Tzourio-Mazoyer et al., 2002). Wernicke's region and its right hemisphere homologue were defined according to the tradition stemming from Geschwind (1970) as the posterior portions of the superior temporal gyri (but see Bogen and Bogen, 1976; Dronkers et al., 2004; Ojemann, 1991 for conflicting views). The superior temporal gyri were again found using Wake Forest University Pickatlas (Tzourio-Mazoyer et al., 2002) and the posterior portions were defined as $Y \leq-20$ in MNI coordinates. The VWFA was defined as a sphere with a $10 \mathrm{~mm}$ radius around the MNI coordinates: $[-43,-54,-12]$ and for the right hemisphere an additional sphere was created around the coordinates: $[43,-54,-12]$. This location has been reported to be the average peak coordinate across 25 word reading studies, with a standard deviation of $5 \mathrm{~mm}$ (McCandliss et al., 2003). See Fig. 6 for an illustration of the three regions.

\section{Results}

\section{Behavioral results}

\section{Accuracy}

Behavioral measures were Bonferroni-corrected for multiple comparisons. A $2 \times 2 \times 2$ factorial repeated-measures ANOVA revealed a main effect of conceptual shift (CS - new vs repeat words, $\mathrm{F}(1,57)=$ 163.4, $\mathrm{p}$ (bonf) $<0.001$ ), a main effect of perceptual modality shift (PS new modality vs same modality, $\mathrm{F}(1,57)=65.1$, $\mathrm{p}$ (bonf) $<0.001)$, a main effect of response shift (RS - new response vs same response, $\mathrm{F}(1,57)=20.8, \mathrm{p}($ bonf $)<0.001)$, several interaction effects: $\mathrm{CS} \times \mathrm{PS}$ $(\mathrm{F}(1,57)=84.6, \mathrm{p}($ bonf $)<0.001), \mathrm{CS} \times \mathrm{RS}(\mathrm{F}(1,57)=84.618$, $\mathrm{p}$ (bonf) $<0.001)$ and PS $\times \operatorname{RS}(\mathrm{F}(1,57)=20.3$, $\mathrm{p}($ bonf $)<0.001)$. No three-way interaction was observed for accuracy $(F(1,57)=1.5$, $\mathrm{p}>0.2$ ). See Fig. 2 .

\section{Response time}

Only correct responses were analyzed. A main effect of $\mathrm{CS}(\mathrm{F}(1,57)=$ 83.02, $\mathrm{p}$ (bonf) $<0.001,93.6 \mathrm{~ms}$ difference) was observed, a main effect of PS $(F(1,57)=167.47, \mathrm{p}$ (bonf) $<0.001,52.2 \mathrm{~ms})$, a main effect of RS $(\mathrm{F}(1,57)=29.8$, $\mathrm{p}$ (bonf) $<0.001,22.9 \mathrm{~ms})$, two two-way interaction effects: $\mathrm{CS} \times \mathrm{RS}(\mathrm{F}(1,57)=212.03, \mathrm{p}($ bonf $)<0.001,62.8 \mathrm{~ms})$ and $\mathrm{PS} \times \mathrm{RS}(\mathrm{F}(1,57)=77.5$, $\mathrm{p}$ (bonf) $<0.001,25.0 \mathrm{~ms})$. We also observed a three-way interaction for response time $(F(1,57)=17.9$, $\mathrm{p}$ (bonf) $<0.001,11.9 \mathrm{~ms})$. No CS $\times$ PS interaction was seen $(\mathrm{F}(1,57)$ $=0.91, \mathrm{p}=0.343,3.1 \mathrm{~ms}$ ). See Fig. 2 .

\section{fMRI results}

\section{Whole brain data}

\section{Main effects}

Conceptual shift (CS). CS was accompanied by increased activation in a network of largely left-lateralized frontal and temporal brain regions including orbitofrontal, inferior frontal (BA 45), premotor, inferior temporal and middle temporal areas (see Fig. 3-top and Table 1). Negative
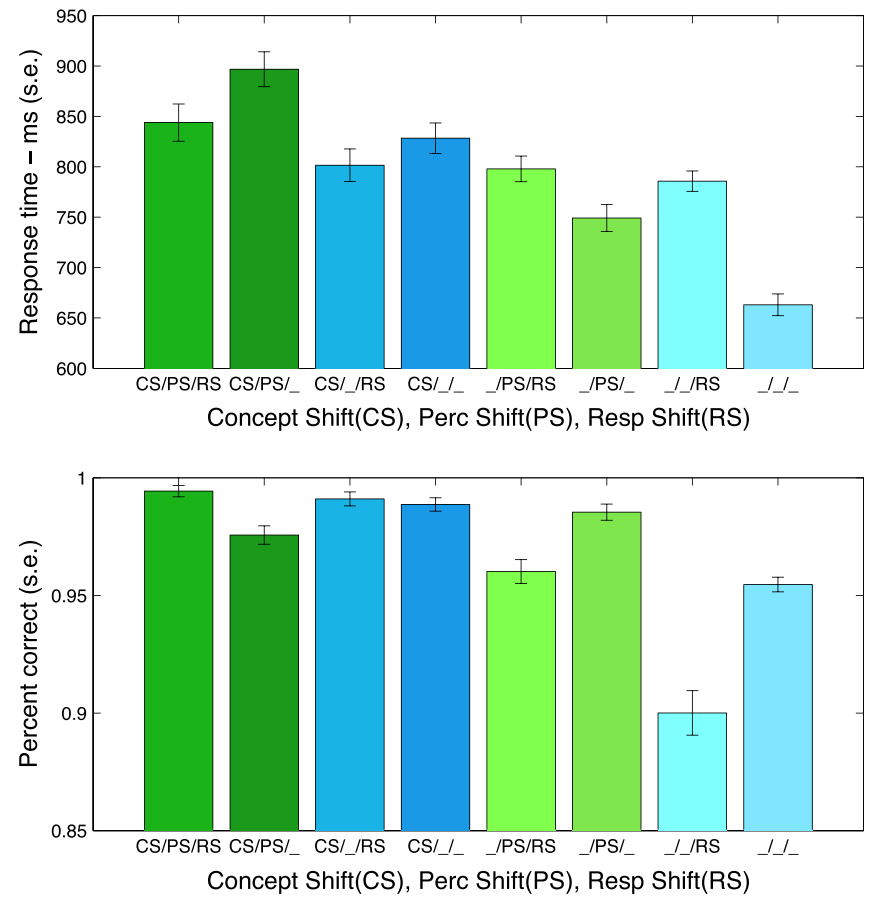

Fig. 2. Behavioral effects. Response time effects of CS (93 ms), PS ( $52 \mathrm{~ms}$ ) and RS ( $23 \mathrm{~ms}$ ) as well as interactions were observed. 


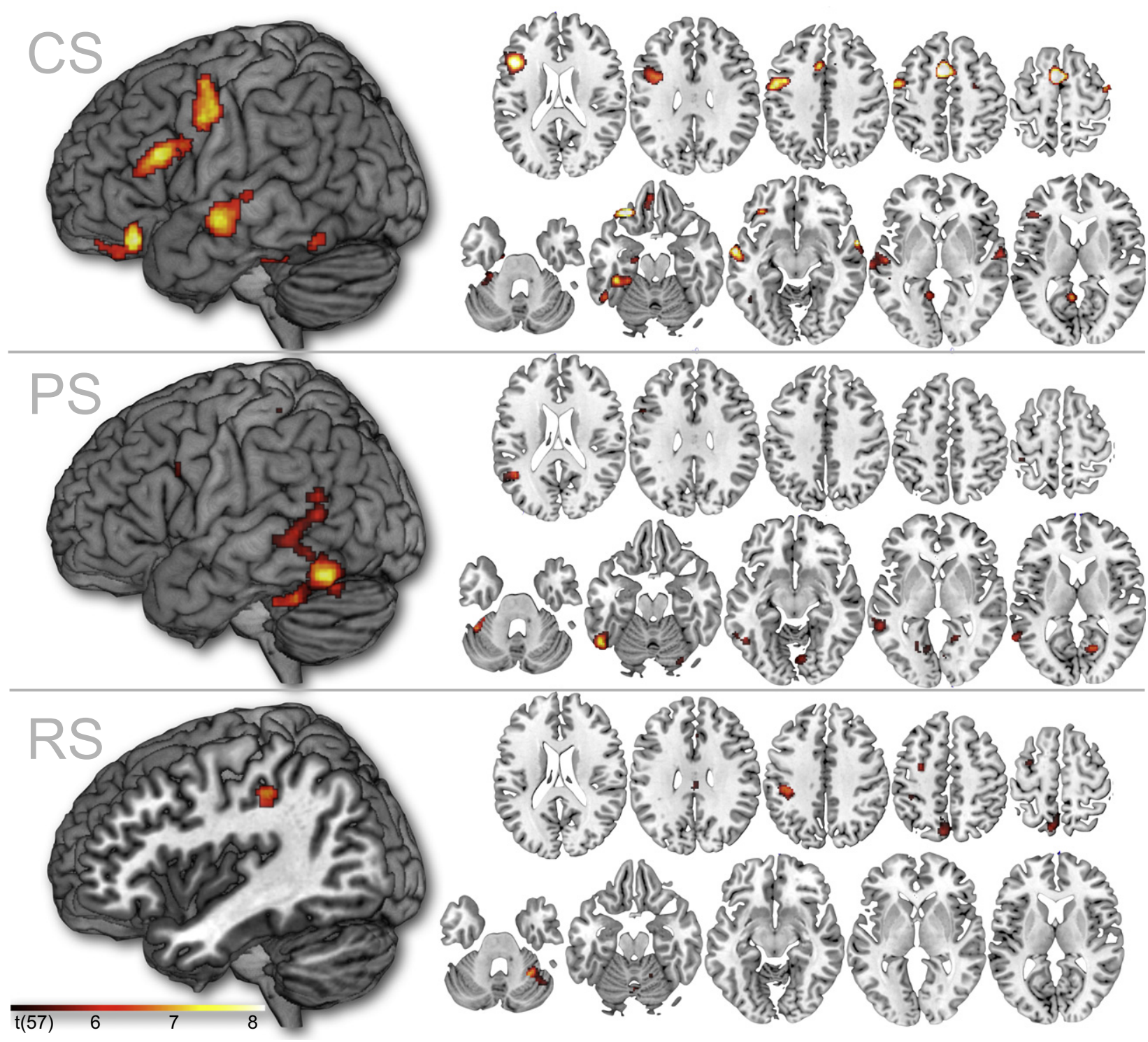

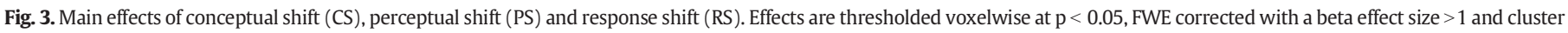
size $>10$.

effects were observed primarily in the parietal lobes, bilaterally, right lateralized in frontal regions including the right inferior frontal region (BA 45) and in the right inferior temporal gyrus (see Table 1).

Perceptual shift (PS). PS was accompanied by primarily left lateralized temporal regions, including inferior and middle temporal regions. But effects were also seen in the left inferior frontal part (see Fig. 3-middle and Table 2). No regions were found to display negative effects of PS.

Response shift (RS). RS was accompanied with activations primarily in motor and parietal regions. A number of midline effects were observed as well (see Fig. 3-bottom and Table 3). No regions were found to display negative effects of RS.

Interactions. A number of two-way interactions were observed at the whole brain level (Fig. 4, Table 4), including both highly significant positive and negative interactions between CS and RS. The negative effect was observed in motor and premotor regions as well as in anterior insula and in the posterior parietal lobe, including precuneus. The negative $\mathrm{CS} \times \mathrm{RS}$ interaction observed reflects regions of the brain that respond more if the current trial is a CS without RS and if the current trial is a nonCS but with RS (i.e. [ - CS / RS + CS / nonRS + nonCS / RS - nonCS / nonRS]). In both cases this involves the presence of a CS in the previous trial. A similar negative interaction between PS and RS was observed. This reflects trials where the previous trial was a PS. Again, effects were observed in the precuneus among other places (Table 4). No 3-way interaction was observed.

Conjunctions. To investigate if Broca's region, Wernicke's region and VWFA are involved in shifts per se or if the processing is specific to conceptual processing we looked for overlapping responses for the three main effects. Here, in contrast to the main effects analysis, we were also concerned about false negatives. We therefore relaxed the statistical threshold to $\mathrm{p}<0.001$, uncorrected for multiple comparisons, and looked for voxels that would be significant at this level over more than one contrast (see Fig. 5).

CSEPS. Similar to the corrected threshold, both Broca's region and VWFA activations were found for both CS and PS at this lower threshold, whereas they were not found for RS. A number of other regions, including Wernicke's region were observed to be shared among CS and PS.

CSERS. Overlaps between CS and RS were observed primarily in the premotor regions, bilaterally and in the supplementary motor region extending into the cingulate. Additional overlap was seen in the retrosplenial region and cerebellum. 
Table 1

Effects of conceptual shift (CS).

\begin{tabular}{|c|c|c|}
\hline Putative anatomical region & Peak MNI & Z-score \\
\hline \multicolumn{3}{|c|}{ Positive effect of conceptual shift [CS-nonCS] } \\
\hline Sup orb. frontal R - BA 11 & $-8,38,-24$ & 5.57 \\
\hline Inf./mid. orb. frontal L - BA 47/11 & $-28,34,-18$ & 7.64 \\
\hline Inf. frontal tri. $\mathrm{L}-\mathrm{BA} 45$ & $-46,18,22$ & 6.90 \\
\hline Supp. motor area L - BA 6 & $-6,10,54$ & Inf. \\
\hline Precentral L -BA 6 & $-42,0,36$ & 6.69 \\
\hline Precentral L - BA 6 & $-50,-2,40$ & 6.43 \\
\hline Precentral R - BA 6 & $60,0,46$ & 5.16 \\
\hline Precentral R - BA 6 & $26,-6,52$ & 5.18 \\
\hline Precentral R - BA 6 & $48,-6,62$ & 6.37 \\
\hline Paracentral lobule L - BA 4/6 & $-8,-30,68$ & 5.22 \\
\hline Postcentral R - BA 4/5 & $16,-38,76$ & 4.99 \\
\hline Sup. temporal R - BA 21 & $62,2,-10$ & 6.52 \\
\hline Sup./mid. temporal L - BA 21 & $-62,-6,-10$ & 6.75 \\
\hline Mid. temporal L - BA 22 & $-68-20,2$ & 5.50 \\
\hline Mid. temporal L - BA 22 & $-50,-36,2$ & 5.14 \\
\hline Parahippocampal L & $-18,-14,-26$ & 6.68 \\
\hline Parahippocampal R & $20,-14,-26$ & 5.51 \\
\hline Retrosplenial/calcarine L - BA 30 & $-6,-52,6$ & 6.34 \\
\hline Fusiform L - BA 20 & $-40,-36,-20$ & 6.25 \\
\hline Inf. temporal L - BA 20 & $-50,-54,-18$ & 5.64 \\
\hline \multicolumn{3}{|c|}{ Negative effect of conceptual shift [nonCS-CS] } \\
\hline Mid orb frontal L - BA 11 & $-22,56,-16$ & 5.27 \\
\hline Sup frontal R - BA 10 & $18,56,24$ & 5.35 \\
\hline Sup frontal R - BA 8 & $12,40,50$ & 5.55 \\
\hline Mid frontal L - BA 10 & $-44,58,4$ & 5.74 \\
\hline Mid frontal $\mathrm{L}-\mathrm{BA} 8$ & $-40,20,44$ & 5.11 \\
\hline Mid frontal R - BA 10 & $44,48,6$ & 6.77 \\
\hline Mid frontal R - BA 46 & $46,40,22$ & 6.30 \\
\hline Mid frontal R - BA 8 & $42,14,44$ & 6.53 \\
\hline Sup medial frontal R - BA 9 & $4,36,38$ & 5.46 \\
\hline Sup medial frontal $\mathrm{R}-\mathrm{BA} 8$ & $8,30,54$ & 5.85 \\
\hline Inf. frontal tri. $\mathrm{R}-\mathrm{BA} 45$ & $54,12,12$ & 7.24 \\
\hline Insula $\mathrm{R}$ & $42,4,2$ & 5.15 \\
\hline Mid temporal R - BA 21 & $62,-44,0$ & 5.70 \\
\hline Inf. temporal R - BA 20 & $64,-44,-24$ & 5.51 \\
\hline Outside brain/inf. parietal L - BA 40 & $-62,-50,44$ & 7.53 \\
\hline Inf. parietal L - BA 40 & $-48,-56,50$ & 7.19 \\
\hline Inf. parietal $\mathrm{R}-\mathrm{BA} 40$ & $56,-34,52$ & Inf. \\
\hline Inf. parietal R - BA 40 & $44,-56,56$ & 7.08 \\
\hline Angular L - BA 39 & $-60,-58,24$ & 6.12 \\
\hline Angular R - BA 7/40 & $40,-64,48$ & 6.80 \\
\hline Precuneus R - BA 7 & $6,-70,42$ & 5.54 \\
\hline Precuneus R - BA 7 & $10,-76,50$ & 5.39 \\
\hline
\end{tabular}

P-FWE $<0.05$, beta $>1$ or $<-1$, cluster $>10$ voxels, peak dist $>12 \mathrm{~mm}$.

PSERS. A large posterior overlap was seen for PS and RS extending over much of the parietal, occipital and posterior temporal lobes as well as cerebellum.

CSEPSERS. Task general shift effects were observed in the premotor cortex, in the retrosplenial/calcarine region and in the cerebellum, all in the left hemisphere.

Table 2

Effects of perceptual shift (PS).

\begin{tabular}{lll}
\hline Putative anatomical region & Peak MNI & Z-score \\
\hline Positive effect of perceptual shift [PS-nonPS] & & \\
Sup med. frontal L - BA 8 & $-2,54,44$ & 4.99 \\
Precentral/inf. front tri. L - BA 6/45 & $-52,12,30$ & 5.02 \\
Postcentral L - BA 4/5 & $-40,-36,60$ & 4.92 \\
Fusiform/inf temporal L - BA 20 & $-42,-44,-28$ & 5.78 \\
Inf temporal L - BA 37 & $-52,-58,-18$ & 6.33 \\
Sup. temporal sulcus L - BA 22/39 & $-44,-54,20$ & 5.72 \\
Retrosplenial/calcarine R - BA 30 & $14,-64,10$ & 5.66 \\
Lingual L - BA 18 & $-20,-60,0$ & 4.97 \\
Lingual R - BA 18 & $8,-74,-10$ & 5.25 \\
Precuneus - BA 7 & $-2,-60,68$ & 4.95 \\
Cuneus - BA 19 & $2,-92,40$ & 5.18 \\
Cerebellum R & $28,-76,-22$ & 5.07 \\
\hline
\end{tabular}

P-FWE $<0.05$, beta $>1$, cluster $>10$ voxels, peak dist $>12 \mathrm{~mm}$.
Table 3

Effects of response shift (RS).

\begin{tabular}{llc}
\hline Putative anatomical region & Peak MNI & Z-score \\
\hline Positive effect of Response shift [RS-nonRS] & & \\
Cingulate ant - BA 32 & $0,28,24$ & 5.08 \\
Cingulate mid - BA 24/32 & $-2,14,34$ & 5.33 \\
Frontal precentral/mid. L - BA 6 & $-28,-6,50$ & 5.40 \\
Parietal inf L - BA 40 & $-40,-30,42$ & 5.83 \\
Cingulum mid. - BA 23 & $0 .-24,32$ & 5.02 \\
Cingulum post. - BA 23 & $0,-38,24$ & 5.01 \\
Precuneus L - BA 7 & $-10,-66,56$ & 5.43 \\
Cerebellum R & $30,-52,-28$ & 6.21 \\
Cerebellum L & $2,-68,-14$ & 5.37 \\
\hline
\end{tabular}

P-FWE $<0.05$, beta $>1$, cluster $>10$ voxels, peak dist $>12 \mathrm{~mm}$.

\section{ROI: Broca's region and right hemisphere homologue}

To investigate lateralization effects and further limit potential false negatives, we investigated the effects of words against the nonmodeled baseline averaged across Broca's region (left inferior frontal gyrus (LIFG), i.e. BAs $44 \& 45$ ) and its right hemisphere homologue (RIFG; see Fig. 6). When comparing average beta-estimates for all conditions in LIFG and RIFG with a paired t-test this revealed a weak non-significant lateralization trend $(t(57)=1.67, \mathrm{P}=0.1) .35 / 58$ participants displayed a left-lateralized effect.

Next, we investigated the lateralization effects for CS, PS and RS. We conducted a $2 \times 2 \times 2 \times 2$ ANOVA ( $\mathrm{CS} \times \mathrm{PS} \times \mathrm{RS} \times$ hemisphere) on these data and, after correcting for multiple comparisons (using Bonferroni correction), we found no significant main effects, but a strong twoway interaction between CS and cerebral hemisphere $(F(1,57)=$ $130.1, \mathrm{p}$ (bonf) $<0.001$ ), a strong interaction between PS and hemisphere $(\mathrm{F}(1,57)=42.5, \mathrm{p}$ (bonf) $<0.001)$, and an interaction between CS and RS $(\mathrm{F}(1,57)=12.854, \mathrm{p}$ (bonf) $<0.02)$. As can be seen from Fig. 6, the regional effects found in Broca's region for CS and PS are countered by negative effects in the right hemisphere homologue of Broca's region, explaining why we find no main effect of CS or PS in the ANOVA and also explaining the strong interaction between hemisphere and the two shift effects. Crucially, no such interaction effect is observed for RS and hemisphere $(F(1,57)=0.3, p>0.5)$, thus limiting the scope of the lateralized shift processing conducted in Broca's area. No other significant effects or interactions were observed in the ROI ANOVA. A lateralization index was computed within the ROI for each contrast separately by a simple left minus right hemisphere subtraction. The lateralization effects for CS and PS were very robust. For CS, 55/58 participants displayed lateralization indices above zero (i.e. left-lateralized); for PS, 46/ 58 participants were found to be left-lateralized, while for RS, 32/58 participants showed a left-lateralized effect. No effects of gender (Wallentin, 2009) were observed for any of the three lateralization indices in Broca's region $(\mathrm{F}(1,57)<1.3, \mathrm{p}$ (uncorr.) $>0.25$ for all three tests).

\section{ROI: Wernicke's region and right hemisphere homologue}

We conducted another $2 \times 2 \times 2 \times 2$ ANOVA ( CS $\times \mathrm{PS} \times \mathrm{RS} \times$ hemisphere) on the average beta estimates for the Wernicke's region ROI and its right hemisphere homologue. We found a significant main lateralization effect $(\mathrm{F}(1,57)=37.96, \mathrm{p}($ bonf $)<0.001)$. No significant main effects of CS, PS or RS when correcting for multiple comparisons ( $p$ (bonf) $>0.05$ for all). We found significant interaction between CS and hemisphere $(\mathrm{F}(1,57)=25.27, \mathrm{p}($ bonf $)<0.001)$ and a significant interaction between PS and hemisphere $(F(1,57)=18.42)$, $\mathrm{p}$ (bonf) $<0.001)$. No interaction between RS and hemisphere was observed $(\mathrm{F}(1,57)=1.67, \mathrm{p}($ bonf $)>0.05)$. We also observed a significant three-way interaction between CS, RS and hemisphere $(F(1,57)=$ $18,09, \mathrm{p}$ (bonf) $<0.001$ ), showing that lateralization is greater for CS in the absence of RS and for NonCS if an RS is present (Fig. 7). No other interactions were observed ( $p$ (bonf) $>0.05$ for all tests). For CS, 47/58 participants displayed a left-lateralized effect, for PS, 41/58 participants were found to be left-lateralized, while for RS, 35/58 participants showed a left-lateralized effect. No effects of gender (Wallentin, 2009) 


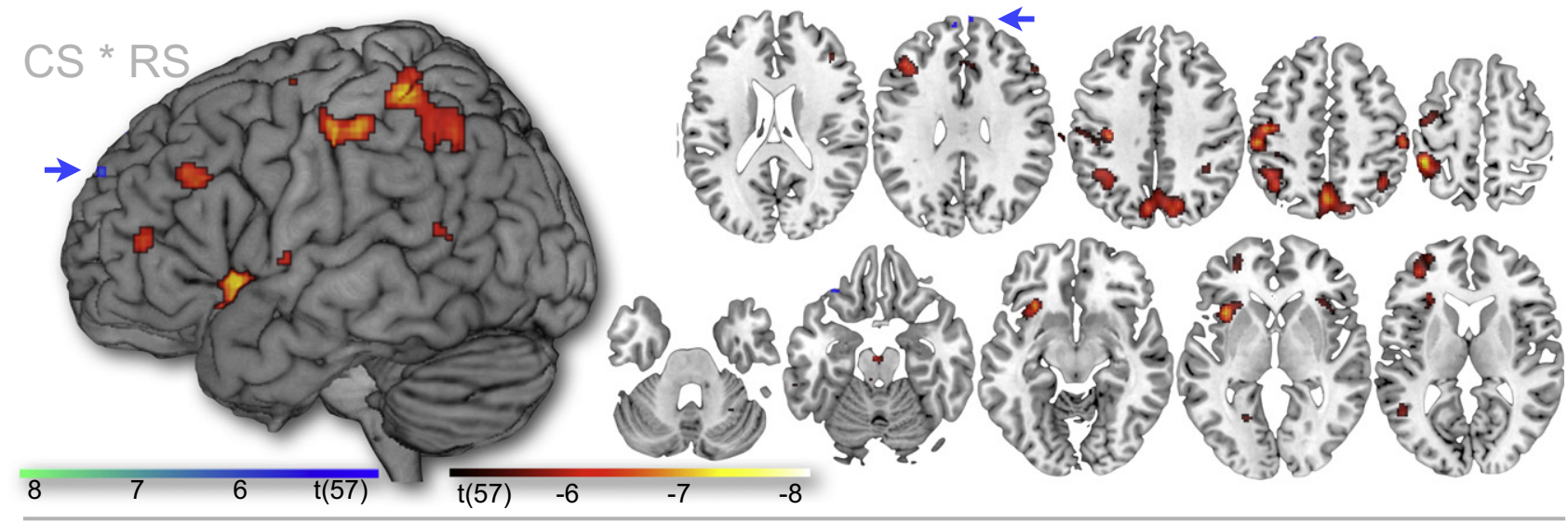

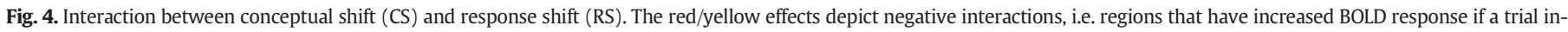

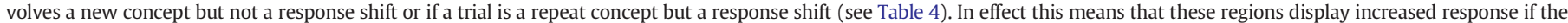
previous trial was a conceptual shift. This may reflect history effects or be a down-stream (venous) response. The map is thresholded voxelwise at $\mathrm{p}<0.05$, FWE corrected.

were observed for any of the three lateralization indices in Wernicke's region $(\mathrm{F}(1,57)<2$, $\mathrm{p}$ (uncorr.) $>0.1$ for all three tests).

ROI: visual word form area and right hemisphere homologue

We again conducted a $2 \times 2 \times 2 \times 2$ ANOVA $(\mathrm{CS} \times \mathrm{PS} \times \mathrm{RS} \times$ hemisphere) on the average beta estimates for the VWFA ROI

Table 4

Interaction effects.

\begin{tabular}{|c|c|c|}
\hline Putative anatomical region & Peak MNI & Z-score \\
\hline \multicolumn{3}{|l|}{ 2-way interactions } \\
\hline \multicolumn{3}{|l|}{ Positive interaction $C S * P S$} \\
\hline \multicolumn{3}{|l|}{ N.S. } \\
\hline \multicolumn{3}{|l|}{ Negative interaction $C S * P S$} \\
\hline \multicolumn{3}{|c|}{$[-C S / P S+C S /$ nonPS + nonCS / PS - nonCS / nonPS $]$} \\
\hline Inf. occipital L - BA 19 & $-40,-68,-10$ & 4.85 \\
\hline Mid. occipital R - BA 39 & $42,-76,26$ & 5.25 \\
\hline \multicolumn{3}{|l|}{ Positive interaction $C S * R S$} \\
\hline \multicolumn{3}{|c|}{ [CS / RS - CS / nonRS - nonCS / RS + nonCS / nonRS] } \\
\hline Frontal sup med L - BA 9 & $-6,60,34$ & 5.36 \\
\hline Frontal sup med $\mathrm{L}-\mathrm{BA} 8$ & $-10,50,50$ & 4.78 \\
\hline Frontal sup med $\mathrm{R}-\mathrm{BA} 8$ & $12,52,48$ & 5.31 \\
\hline Frontal inf orb $\mathrm{L}-\mathrm{BA} 47$ & $-32,32,-18$ & 5.19 \\
\hline Rectus/midline & $0,36,-28$ & 5.14 \\
\hline \multicolumn{3}{|l|}{ Negative interaction $C S * R S$} \\
\hline \multicolumn{3}{|c|}{$[-C S / R S+C S /$ nonRS + non $C S / R S-$ nonCS / nonRS $]$} \\
\hline Frontal mid L - BA 46 & $-36,46,12$ & 5.59 \\
\hline Frontal mid L - BA 46 & $-40,28,30$ & 5.63 \\
\hline Frontal mid R - BA 46 & $40,36,22$ & 5.31 \\
\hline Frontal sup L - BA 6 & $-14,-8,76$ & 5.28 \\
\hline Cingulum mid R - BA 24 & $6,30,32$ & 5.09 \\
\hline Insula L - BA 13 & $-36,14,-4$ & 6.09 \\
\hline Insula $\mathrm{L}-\mathrm{BA} 13$ & $-28,28,10$ & 5.25 \\
\hline Insula $\mathrm{R}-\mathrm{BA} 13$ & $32,24,4$ & 5.38 \\
\hline Insula R - BA 13 & $40,20,0$ & 4.9 \\
\hline Rolandic oper L - BA 13 & $-50,-2,4$ & 5.46 \\
\hline Postcentral R BA 2/3/4 & $-36,-18,44$ & 6.43 \\
\hline Postcentral R BA 2/3/4 & $54,-26,50$ & 5.68 \\
\hline Temporal inf $\mathrm{L}$ & $-62,-36,-16$ & 5.06 \\
\hline Temporal mid L - BA 21/22 & $-50,-52,16$ & 5.41 \\
\hline Parietal inf $\mathrm{L}-\mathrm{BA} 40$ & $-42,-42,60$ & 6.07 \\
\hline Parietal inf $\mathrm{R}-\mathrm{BA} 40$ & $40,-56,48$ & 5.63 \\
\hline Precuneus/midline - BA 7 & $0,-68,46$ & 6.21 \\
\hline Precuneus $\mathrm{R}-\mathrm{BA} 7$ & $16,-72,40$ & 5.54 \\
\hline Brain stem & $-2,-16,-20$ & 5.26 \\
\hline \multicolumn{3}{|l|}{ Positive interaction $P S * R S$} \\
\hline \multicolumn{3}{|l|}{ N.S. } \\
\hline \multicolumn{3}{|l|}{ Negative interaction $P S * R S$} \\
\hline \multicolumn{3}{|c|}{$[-P S / R S+P S /$ nonRS + nonPS / RS - nonPS / nonRS $]$} \\
\hline Parietal inf L - BA 40 & $-30,-56,46$ & 5.02 \\
\hline Putamen R & $28,20,-2$ & 4.90 \\
\hline
\end{tabular}

P-FWE $<0.05$, peak dist $>12 \mathrm{~mm}$.
(Dehaene and Cohen, 2011) and its right hemisphere homologue. We found a nonsignificant main lateralization effect $(F(1,57)=4.13$, $\mathrm{p}($ bonf $)>0.05)$. We found significant main effects of $\mathrm{CS}(\mathrm{F}(1,57)=$ 11.67, $\mathrm{p}$ (bonf) $<0.02)$ and of PS $(\mathrm{F}(1,57)=12.6, \mathrm{p}($ bonf $)<0.02)$ but no main effect of $\operatorname{RS}(F(1,57)=0.18, p($ bonf $)>0.05)$. We also found significant interactions between $\mathrm{CS}$ and hemisphere $(\mathrm{F}(1,57)=30.75$, $\mathrm{p}($ bonf $)<0.001)$ and between PS and hemisphere $(\mathrm{F}(1,57)=18.92$, $\mathrm{p}($ bonf $)<0.001)$. No interaction for RS and hemisphere was observed $(\mathrm{F}(1,57)=2.5, \mathrm{p}($ bonf $)>0.05)$ and no other significant interactions were observed ( $\mathrm{p}$ (bonf) $>0.05$ for all tests). For CS, $45 / 58$ participants displayed a left-lateralized effect, for PS, 43/58 participants were found to be left-lateralized, while for RS, 28/58 participants showed a left-lateralized effect. No effects of gender (Wallentin, 2009) were observed for any of the three lateralization indices in VWFA $(\mathrm{F}(1,57)<03, \mathrm{p}$ (uncorr. $)>0.6$ for all three tests).

\section{Correlation between lateralization indices}

In order to investigate the relationship between the different lateralization indices, we made a Pearson correlation matrix (see Table 5). Lateralization for the different tasks was found not to be correlated within brain regions. However, a positive correlation within the PS task was found between both Broca's region, Wernicke's region and VWFA (at uncorrected significance levels) and a similar correlation was found for CS, however only between Broca's region and the two other regions and not between Wernicke's region and VWFA. CS in Broca's region was also found to be positively correlated to RS in Wernicke's region. No positive correlations were found for RS across regions.

\section{Discussion}

\section{Broca's region}

We found that conceptual shifts (CS) and perceptual shifts (PS) but not response shifts (RS) in relation to a linguistic one-back task resulted in a strongly left-lateralized BOLD response in Broca's region (LIFG; BAs $44 \& 45$ ). Word stimuli as such (relative to baseline) did not yield a significantly lateralized effect in Broca's region (Fig. 6, top). Increased processing costs were observed in terms of prolonged response times for all types of task shifts, but only CS (peak coordinates: $-46,18,22$ ) and PS (peak coordinates: 52, 14, 30) yielded significant leftlateralized Broca's region responses (see Tables 1 and 2 and Figs. 3, 5 and 6). This demonstrates that shift cost effects in this region are not task-general, but limited to certain processes, most likely those that are conceptual in nature. Both PS and nonPS conditions contain CS trials to an equal amount due to randomization of the stimuli within and 

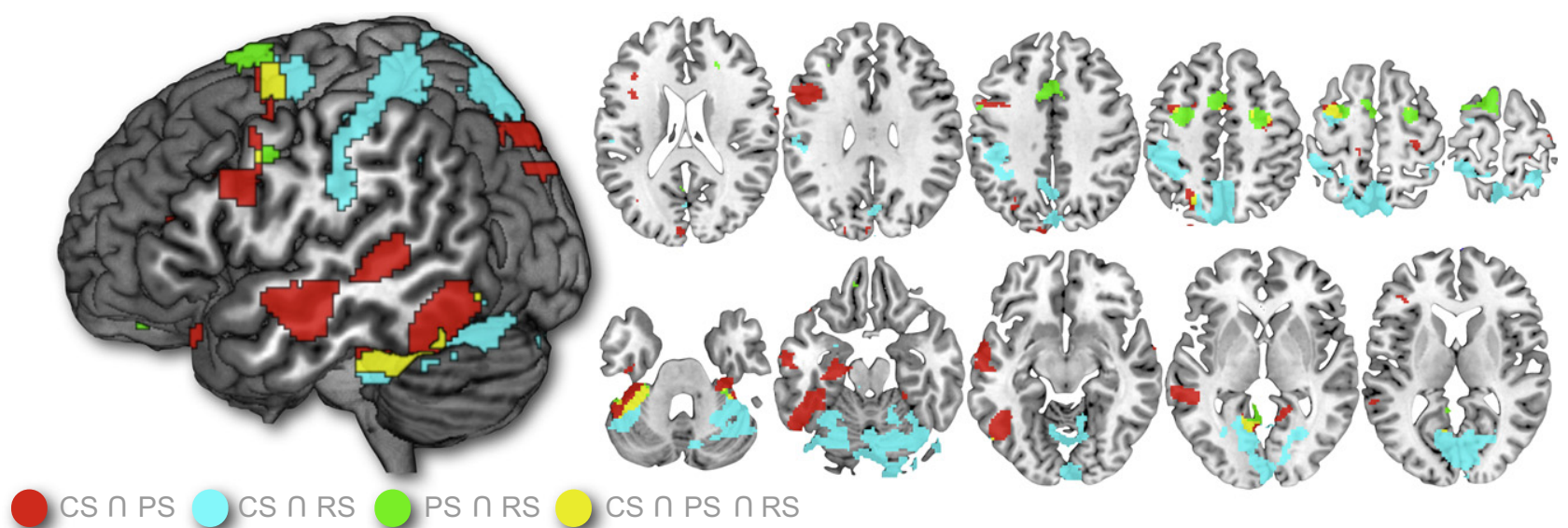

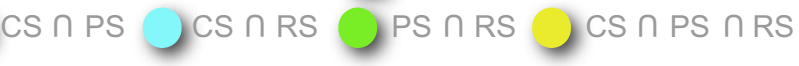

Fig. 5. Conjunctions. Red: conceptual shift (CS) \& perceptual shift (PS). Green: CS \& response shift (RS). Cyan: PS \& RS. Yellow. CS \& PS \& RS. Each effect was thresholded at p $<0.001$, uncorrected, to balance false positives and false negatives. Unspecific shift costs are primarily seen in premotor and cerebellar regions, suggesting a link to motor output. More widespread overlap is seen for conceptual shift and perceptual shift in frontal and temporal regions, including Broca's area, Wernicke's region and VWFA. No effect for RS is seen in either region, indicating that it is not task shifts as such, but more language related shifts that evoke responses here.
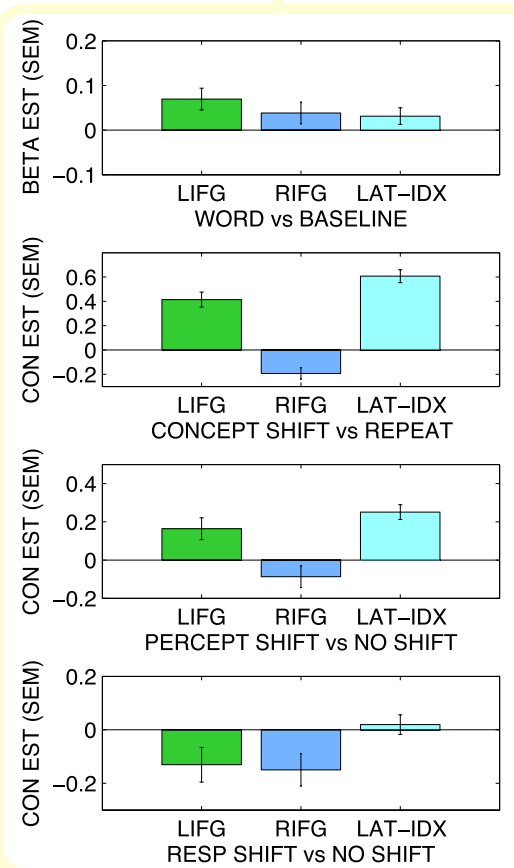
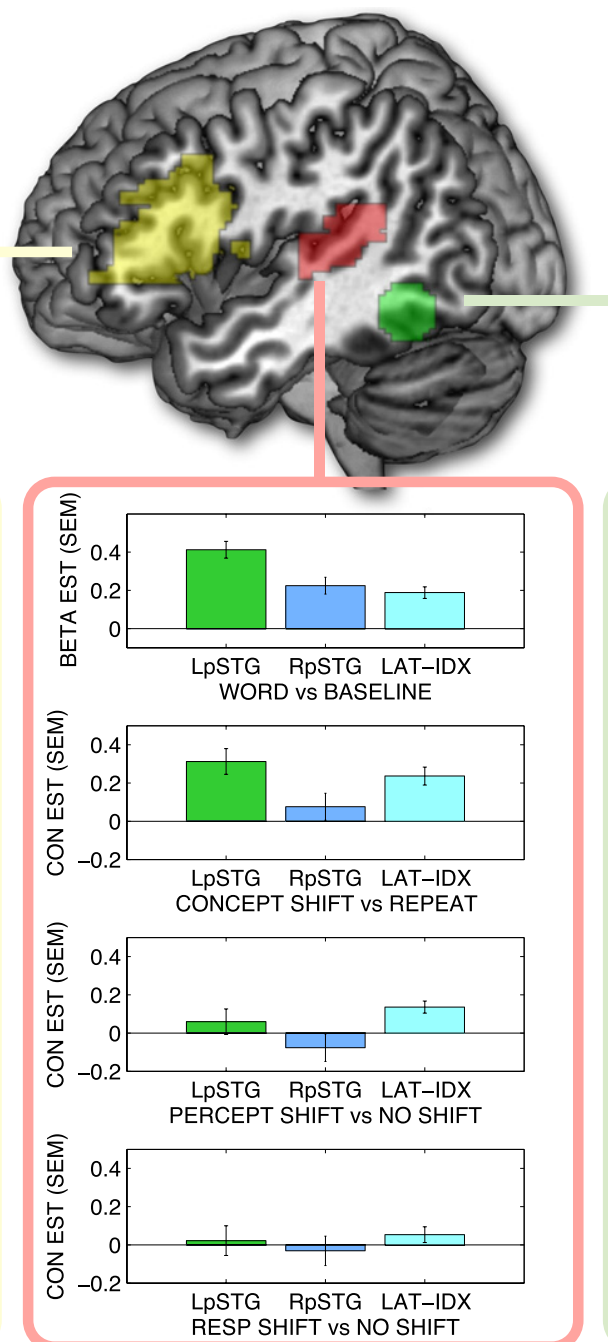
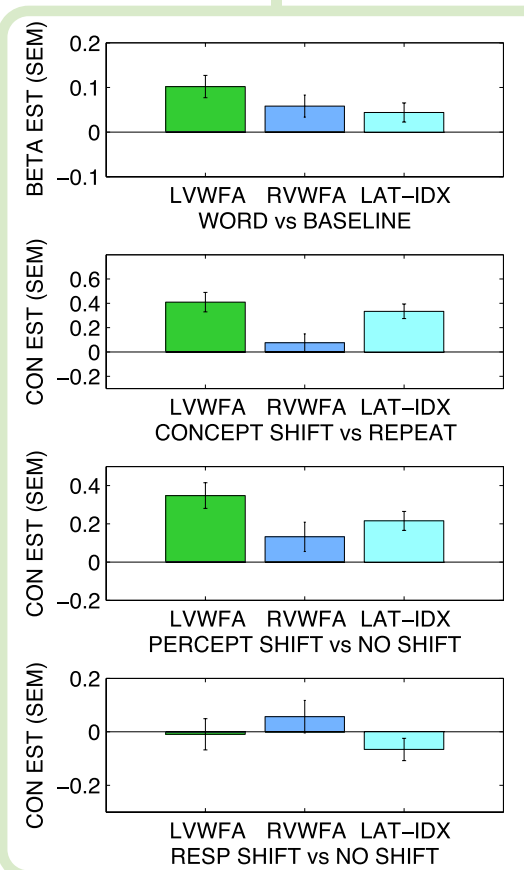

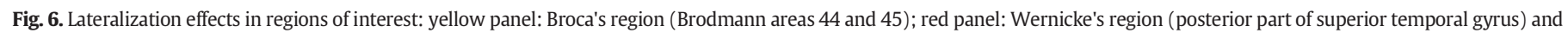

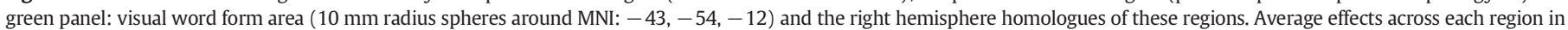

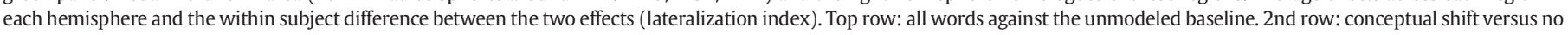
shift. 3rd row: perceptual modality shift versus no shift. 4th row: response shift versus no shift. 

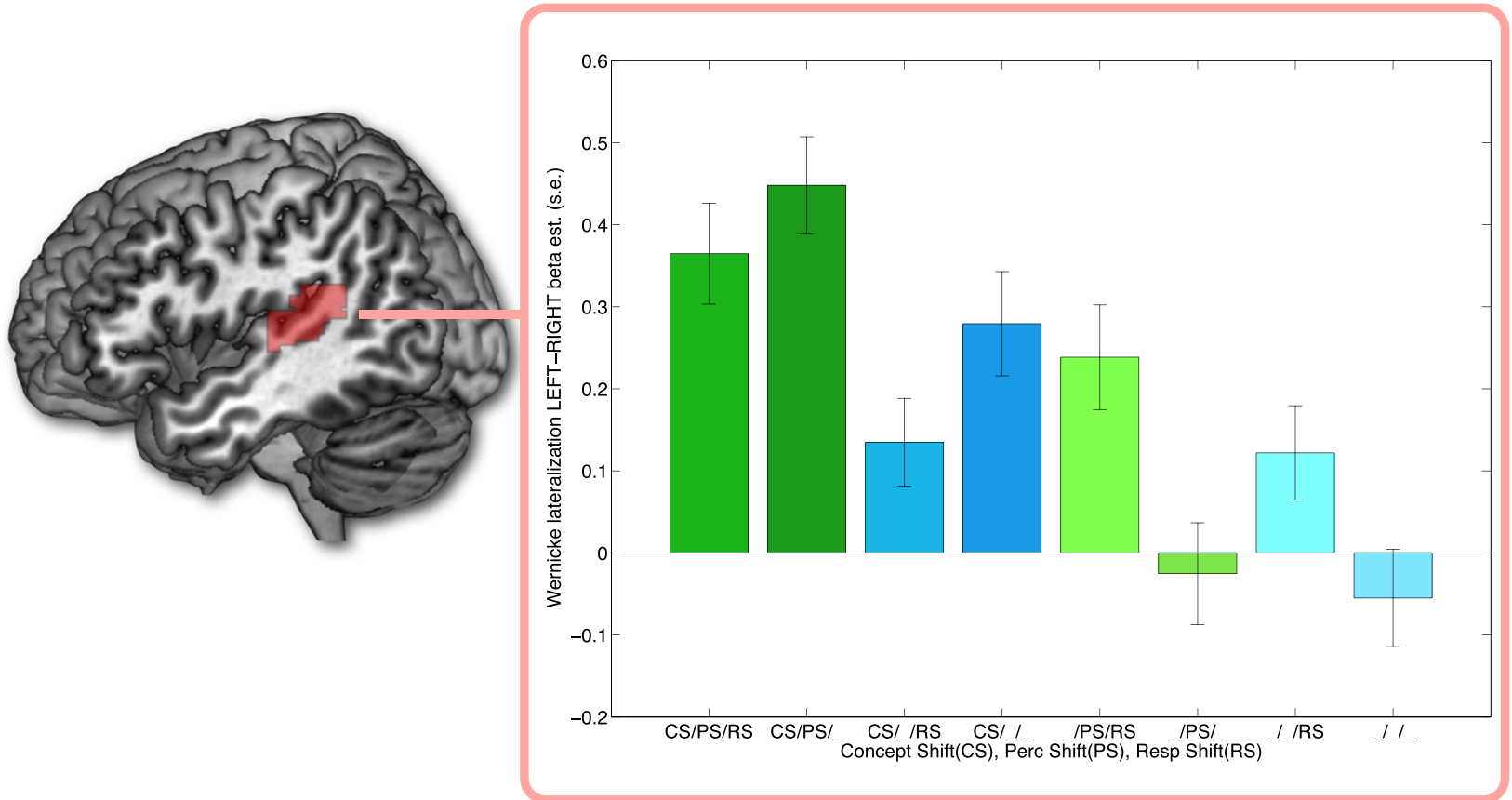

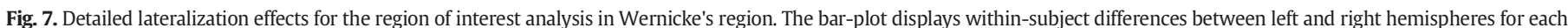

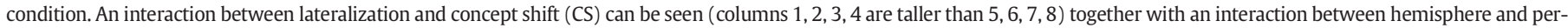

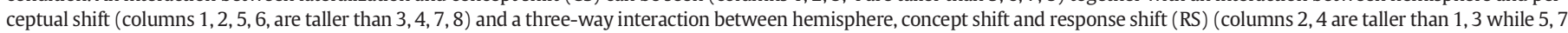

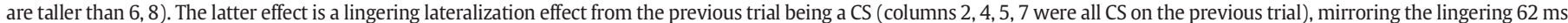
response time costs observed for trials that were CS on the previous trial.

across participants. The PS effect (PS-nonPS) therefore cannot be said to reflect CS. However, PS trials are more conceptual than nonPS trials in the sense that the one-back task within perceptual modality can be solved based solely on perceptual surface features whereas a word comparison across modalities requires a semantic representation or at least a translation/recoding of linguistic features (letters to phonemes or vice versa). One might argue that a beneficial task solving strategy would be to encode all trials at the conceptual level. In that case, however, one would not expect to see any difference between PS and nonPS. This is not supported by data. Both the fMRI data and the response time pattern clearly show that PS trials are more costly to process than nonPS trials, suggesting a difference in strategy for solving the two trial types with PS perhaps relying more on conceptual content than nonPS.

A response shift, on the other hand (going from a "repeat" response to a "new" response or the other way), does not on average have any added conceptual content compared to a non-shift (going from "repeat" to "repeat" or from "new" to "new"). Task general shift effects were

Table 5

Lateralization across tasks and regions.

\begin{tabular}{lcccccccc}
\hline Lateralization & CS-Br & $\begin{array}{l}\text { CS- } \\
\text { We }\end{array}$ & $\begin{array}{l}\text { CS- } \\
\text { VW }\end{array}$ & PS-Br & $\begin{array}{l}\text { PS- } \\
\text { We }\end{array}$ & $\begin{array}{l}\text { PS- } \\
\text { VW }\end{array}$ & $\begin{array}{l}\text { RS- } \\
\text { Br }\end{array}$ & $\begin{array}{l}\text { RS- } \\
\text { We }\end{array}$ \\
\hline CS Broca & & & & & & & & \\
CS Wernicke & $\mathbf{0 . 3 8}^{* *}$ & & & & & & & \\
CS VWFA & $\mathbf{0 . 2 6}^{*}$ & 0.23 & & & & & & \\
PS Broca & 0.11 & 0.13 & -0.10 & & & & & \\
PS Wernicke & 0.10 & 0.07 & 0.17 & $\mathbf{0 . 3 2}^{*}$ & & & & \\
PS VWFA & 0.16 & 0.08 & -0.24 & $\mathbf{0 . 3 9}^{* *}$ & $\mathbf{0 . 2 9}^{*}$ & & & \\
RS Broca & 0.11 & 0.13 & 0.08 & -0.01 & 0.13 & -0.02 & & \\
RS Wernicke & $\mathbf{0 . 3 2}$ & 0.13 & 0.08 & -0.09 & -0.06 & 0.11 & 0.08 & \\
RS VWFA & -0.24 & 0.11 & 0.06 & -0.24 & -0.14 & -0.12 & 0.08 & -0.01
\end{tabular}

Pearson correlation between lateralization indices for conceptual shift (CS), perceptual shift (PS) and response shift (RS) in Broca's region, Wernicke's region and visual word form area (VWFA).

Significant correlations are written in bold.

$* \mathrm{p}<0.05$ uncorrected.

** $\mathrm{p}<0.005$ uncorrected. observed more dorsally in premotor cortex (Fig. 4), consistent with a prolonged response time.

The lateralization for the CS contrast was not only visible in the left hemisphere. The CS-nonCS contrast yielded a negative effect in the right hemisphere homologue of Broca's region both in the whole brain data and in the ROI analysis, suggesting that this effect does not originate in one hemisphere but reflects a genuine lateralization of processes (Fig. 6, Table 1). For the PS contrast this effect could also be observed in Broca's region, however only in the ROI analysis (see Fig. 6).

The regions observed for CS are overlapping with those observed in studies of other types of linguistic manipulations, such as semantic integration errors (Christensen and Wallentin, 2011), semantic competition (January et al., 2009; Schnur et al., 2009; Snyder et al., 2007), syntactic complexity (Christensen and Wallentin, 2011; Christensen et al., 2013; Kristensen et al., 2013; Tettamanti et al., 2009), syntactic working memory (Fiebach et al., 2005) and linguistic working memory in general (Kristensen and Wallentin, in press; Wallentin et al., 2006). Wallentin et al. (2006) found that Broca's region activity correlated with response time in a working memory task where a visual scene was probed by linguistic cues. This finding was recently replicated (Kristensen and Wallentin, in press) in a reanalysis of a study of working memory for previously read sentences probed with linguistic cues (Wallentin et al., 2008). Again, Broca's region activity was found to be correlated with response time, suggesting that increased linguistic working memory demands yield increased Broca's region activation. In their study of semantic and syntactic manipulations, Christensen and Wallentin (2011) found that Broca's region activity was also correlated with response time in a sentence acceptability judgment task, and crucially, in this experiment responses were only collected on every other task, making the neural effect independent of whether a response was given or not. This demonstrates that this is not a motor effect. Broca's region activation has also been observed for linguistic comprehension where no overt responses were produced, such as for increased emotional intensity during listening to a narrative (Wallentin et al., 2011).

Together, the above-mentioned findings and those from the present experiment are compatible with theories suggesting that Broca's region 
is involved in processing linguistic processing costs (Kristensen and Wallentin, in press). They may also to a certain extent be compatible with theories positing a role for Broca's region in cognitive control (Novick et al., 2010). However, a key limitation demonstrated by the present results, is that the region is not involved in processing all kinds of cognitive control. Some conceptual content may be needed in order for Broca's region to become activated.

The present study includes a relatively large number of participants $(n=58)$ and a large number of trials per participant (400). This ensures that our results are robust and replicable. Whole brain effects are corrected at the family wise error level for multiple comparisons and for main effects (CS, PS and RS) effects are further thresholded at a beta-estimate level larger than one (at 2 nd level). This ensures that the large number of participants do not lead to significant, but negligible effect sizes. Subsequently, we conducted two more liberal analyses in an attempt to rule out false negatives. These show that the lateralization effect for CS in Broca's region is present in 95\% of our participants, a distribution similar to that seen in aphasia studies (Pedersen et al., 1995) and in sodium amytal (Wada) tests of cerebral speech dominance (Rasmussen and Milner, 1977; Woermann et al., 2003). The degree to which lateralization indices found in the present experiment can be predictive of aphasia symptoms remains to be studied.

We have focused our Broca's region analyses on Brodmann areas 44 and 45. In our ROI analysis, we do not distinguish between these two regions. This is based on our prior findings that the effect observed with linguistic fMRI contrasts are usually located close to the border of BAs 44 and 45, extending into both regions (e.g. see Kristensen and Wallentin, in press for a review). Apart from the BA 44/45 effect, however, we also see a strong effect in BA 47 for CS. BA 47 has recently been shown to be structurally dissimilar from BAs 44 and 45 (Amunts and Zilles, 2012) and has, based on this, been suggested also to be functionally separate. Meta-analyses have pointed towards a role for BA 47 in semantic processing (Binder et al., 2009), which would be consistent with our findings. In the processing of polyrhythms (Vuust and Roepstorff, 2008), an increase in BA 47 has also consistently been observed, both for listening to and for producing polyrhythms compared to simple rhythms (Vuust et al., 2006, 2011). Interestingly, polyrhythm is a strong example of a cognitive conflict (Novick et al., 2010) where musicians play one rhythm (e.g. a 3/4 beat) on top of another rhythmic meter (e.g. a $4 / 4$ beat). This imposes a strong sense of tension both in the listener and in the performer. This would suggest that this region is involved in the processing of cognitive conflicts, both at the conceptual (in the CS contrast) and at the non-conceptual level (as with music). This interpretation, however, would lead to the prediction that PS and RS should also evoke activation in BA 47, which we do not see in this study. At present, we are therefore not able to make a coherent interpretation of the pattern of activations observed in BA 47. Studies that separate cognitive conflict and semantic load are needed to shed light on these apparent inconsistencies. But we note that the results in the present study are very strong with a z-score of 7.6 in BA 47. We are therefore confident that the BA 47 effect is not a false positive and further that the observed lack of results for PS and RS probably do not reflect false negatives either, as we allowed a search for effects using a lowered statistical threshold in our conjunction analysis.

\section{Wernicke's region and visual word form area}

Apart from the described results in Broca's region, the main effect of perceptual shift (PS) yielded a predominantly posterior network of activations (Fig. 3, Table 2). These were located in the ventral and superior part of the temporal lobe. The ventral region was overlapping with the visual word form area (Dehaene and Cohen, 2011; McCandliss et al., 2003), while the superior regions were in the vicinity of Wernicke's region, argued by Wernicke (1874) to be the seat of auditory sound images ("Klangbilder"), i.e. the auditory equivalent of the visual word forms. Since PS involves a translation from visual word forms to auditory word forms or vice versa, it makes sense that both regions are involved in this process. Our findings are thus consistent with the idea that these regions are involved in modality specific processing of words and that this effect is enhanced when the stimuli need to be recoded into a new modality. With the lowered threshold and in the ROI analyses we also saw an effect of CS in both regions.

Similar to the effect in Broca's region, activation in Wernicke's region and VWFA were found to be highly left lateralized, although the lateralization effects did not reach as high as CS did in Broca's region.

In Wernicke's region $81 \%$ were found to be left lateralized in the CS task and $71 \%$ in the PS task. In VWFA 78\% were found to be left lateralized in the CS task and 74\% in the PS task. Another difference was that while the lateralization in Broca's region was composed of both an increase of activity in the left hemisphere and a decrease in activity in the right hemisphere (Fig. 6) for CS and PS, this was only the case for PS in Wernicke's region and for neither CS nor PS in VWFA. Both left and right hemispheres were found to yield positive responses to CS on average and the lateralization thus came about as a relative difference in positive responses (see Fig. 6).

\section{Lateralization across regions and tasks}

When comparing lateralization across tasks and regions of interest, it was found that lateralization indices for Broca's region, Wernicke's region and VWFA were correlated within tasks whereas lateralization for the different tasks was not correlated within regions (see Table 5). Thus, although both task contrasts produce highly lateralized effects, these may not be directly comparable. One previous study found that the hemispheric lateralization of the VWFA was correlated with the lateralization of the LIFG (Cai et al., 2010). In our study we thus replicate this result, but crucially, only within task.

Apart from the fact that the BOLD signal is a composite measure that depends on a number of factors other than brain activity (Gusnard and Raichle, 2001; Logothetis, 2008), the lack of correlation across tasks suggests that lateralization may be a complex phenomenon where an individual may show more or less lateralization for different conceptual tasks, depending on e.g. language proficiency and prior experience. Differences in lateralization for the PS contrast may thus reflect reading abilities and reading strategies whereas lateralization for CS may reflect other more or less orthogonal skills, such as vocabulary size. Other language tasks may display different lateralization patterns and this leads to the hypothesis that different brain regions within the language network may be differently linked for different tasks and that these links form functional specialization rather than individual brain regions by themselves. Further studies are needed in order to test these hypotheses.

Lateralization has been found to correlate with handedness, both when studied using fMRI (Szaflarski et al., 2002) and functional transcranial Doppler sonography (Knecht et al., 2000a, 2000b). In this study we only included right-handed participants. We therefore cannot say whether the observed lateralization effects would show different relationships to handedness, although it seems likely.

\section{Two-way and three-way interactions}

Both in the behavioral and in the neuroimaging data a strong interaction between CS and RS and between PS and RS was found. The CS $\times$ RS interaction reflects increased processing costs if the current trial is a CS without RS or if the current trial is a repeat (nonCS) but with RS. In both cases this involves a CS being present in the previous trial. This effect is clearly visible in the response time data (Fig. 2, top) where columns 2 and 4 represent CS in the absence of RS and columns 5 and 7 represent RS in the absence of CS. These columns are all taller than their direct counterparts (columns 1, 3,6 and 8). This means that there is a lingering response time cost of almost $63 \mathrm{~ms}$ if the previous trial was a CS. The PS $\times$ RS interaction reflects a similar lingering processing cost $(25 \mathrm{~ms})$ if the previous trial was a perceptual shift. Such 
short-term dependencies in response times are well-known (Kelly et al., 2001; Laming, 1979; Spivey, 2007; Van Orden et al., 2003), but are not, to our knowledge studied in an fMRI context. In the ROI analysis we see a CS $\times$ RS interaction in Broca's region, but when looking at the more conservative whole brain results we see that the peak activations are outside Broca's region, suggesting that the ROI result may be a spillover effect from neighboring regions (insula, middle frontal gyrus see Table 4, Fig. 4). The whole brain effects also reveal that the anterior insula effect has a bilateral distribution (although more pronounced towards the left, see Fig. 4; Table 4). Crucially, the CS $\times$ RS interaction was not found to be lateralized in the ROI analysis, i.e. no three-way interaction $(\mathrm{CS} \times \mathrm{RS} \times$ hemisphere) was observed $(\mathrm{F}(1.57)=0.002$, $\mathrm{p}$ (bonf) $=1)$. We therefore conclude that the effect from the previous CS trial in Broca's region is not similar to the effect observed for the current CS trial.

In Wernicke's region, however, this exact three-way interaction (CS $\times \mathrm{RS} \times$ hemisphere) was observed in the ROI analysis (Fig. 7). In the posterior temporal area we thus in addition to the increased lateralization for CS and PS in isolation find an effect of the preceding trial. If the current trial is a CS then we find greater lateralization if the trial does not include a response shift (nonRS), indicating that the previous trial was also a CS (columns 2 and 4 vs. 1 and 3 in Fig. 7). If, however, the current trial is a nonCS, then we find greater lateralization if the trial is also an RS, i.e. again indicating that the previous trial was a CS (columns 5 and 7 vs. 6 and 8 in Fig. 7).

If the brain thus has a BOLD equivalent of the history effects observed in the behavioral data, our findings suggest that it originates from Wernicke's region rather than Broca's region. One problem with this type of analysis in fMRI is the poor temporal resolution of the BOLD response. An interpretation of the regional interaction effects found in the whole brain analysis could be that at least some of them reflect regions with a delayed BOLD response from the previous trial in regions with a different response function from the standard hemodynamic response function incorporated in this analysis. Anterior insula has been found to have a slower HRF, similar to that of a down-stream venous signal (Gonzalez-Castillo et al., 2012; Kruggel and von Cramon, 1999a, 1999b). In theory, the insula signal thus might reflect previous activity in Broca's region (or elsewhere), reaching the veins in insula with a 2-5 s delay (the intertrial interval in the current experiment) compared to a cortical signal. Similarly, we see interaction effects in posterior brain regions, such as the precuneus (see Tables 3-4). This region also holds large draining veins that may transport blood from posterior perceptual regions. Again, we may be dealing with a down-stream effect of the previous trials (Kruggel and von Cramon, 1999a, 1999b). Further studies are needed to elucidate these claims.

\section{Repetition suppression and prediction error}

Task shift effects reflect the inverse of what is known as repetition suppression, i.e. the fact that the neural responses observed for various stimuli are often found to be attenuated when repeated (Dehaene et al., 2001; Orfanidou et al., 2006; Raichle et al., 1994; Schacter and Buckner, 1998; Summerfeld et al., 2011; Summerfield et al., 2008; Todorovic et al., 2011). Repetition suppression has been interpreted as an instance of predictive neural coding and predictive coding has been suggested as an overarching framework for how the brain works (Clark, 2013; Friston, 2010). In this sense task shift effects may be hypothesized to reflect a prediction error in relation to a kind of status quo bias. Such a bias has been also observed in relation to certain cognitive tasks (e.g. Kahneman et al., 1991; Nicolle et al., 2011). Our results may thus be interpreted to reflect a very simplistic kind of prediction error, working on a trial by trial basis. Predictive coding within cognition and language, however, of course have to work on multiple timescales from phoneme prediction via words to prediction of word order and pragmatic content (Levy, 2008). Across the present experiment, for example, the CS words were more frequent than the nonCS words (250/150) and in the frame of the whole experiment, the CS trials should thus yield less prediction error than nonCS words. This effect would work in the opposite direction of the trial-by-trial shift effect. Similarly, a CS or PS following another CS or PS should be less costly. In fact we see the opposite. There is a lingering response time penalty for a CS following a CS (62 $\mathrm{ms}$ ) and for a PS following a PS ( $25 \mathrm{~ms})$. This history effect is also present in the neuroimaging data in the widespread CS $\times$ RS and PS $\times$ RS interactions (see above). In the ROI analysis of Wernicke's region we additionally find the history effect in lateralization where the lateralization level is found to reflect the nature of both the current and the previous trials (Fig. 7). These effects are difficult to reconcile with a priming or prediction error approach. It is therefore not clear how far an interpretation of the activity in the investigated regions as reflecting a more general linguistic prediction error can be taken.

Adding to this complexity is the observation of a widespread set of deactivations, including parietal and right hemisphere regions, as a function of conceptual shift (i.e. regions that are more active during non-shifts than during shifts - see Table 1). These effects are also hard to reconcile within a framework where prediction error is the main driver of neural responses. Considering the memory components of the current experiment, the main difference between CS and nonCS conditions is in the comparison between the previous word (encoded in short-term memory) and the incoming word. In the CS condition there is a mismatch between these two items plus the current word has to be encoded for the next trial. In nonCS trials this is not necessary as it was already encoded during the previous trial. It is thus difficult to see that there are cognitive operations in the nonCS condition that are not present in the CS, except for recognition. But given that the whole priming literature suggests that recognition is primarily linked to decreases in activation, it seems difficult to argue why it should be responsible for the widespread activations that we see as a negative effect in the CS-nonCS contrast. It becomes even more complicated, since the parietal regions found to be deactivated as a function of CS to a large extent are the same as those that are found in the $\mathrm{CS} \times \mathrm{RS}$ interaction (Fig. 4, Table 4). This means that these regions are not only less activated if the current trial is a CS but they are also more activated if the previous trial was a CS. This type of strange switch behavior is intriguing but very hard to interpret. Further studies are needed to investigate if this effect is real or somehow linked to issues related to the sluggish nature of the BOLD response.

\section{Conclusion}

We conclude that Broca's region (BAs 44 \& 45) activation and lateralization for task shift effects is very strong, and selective for task shift effects with a conceptual content. Similar effects are observed in Wernicke's region and the visual word form area, and lateralization is correlated across regions, however only within tasks. Lastly, response time and lateralization in Wernicke's region not only reflect trial type, but also trial history.

\section{Acknowledgments}

This paper was supported by the MindLab grants from the Danish Ministry of Science, Technology and Innovation. The authors would like to thank Gustavo Sudre for giving access to the stimuli.

\section{References}

Amunts, K., Zilles, K., 2012. Architecture and organizational principles of Brocas's region. Trends Cogn. Sci. 16, 418-426.

Ashburner, J., Friston, K.J., 2005. Unified segmentation. NeuroImage 26, 839-851.

Ben-Shachar, M., Palti, D., Grodzinsky, Y., 2004. Neural correlates of syntactic movement: converging evidence from two fMRI experiments. Neurolmage 21, 1320-1336.

Bethmann, A., Tempelmann, C., De Bleser, R., Scheich, H., Brechmann, A., 2007. Determining language laterality by fMRI and dichotic listening. Brain Res. 1133, 145-157.

Binder, J.R., Frost, J.A., Hammeke, T.A., Rao, S.M., Cox, R.W., 1996. Function of the left planum temporale in auditory and linguistic processing. Brain 119, 1239-1247. 
Binder, J.R., Frost, J.A., Hammeke, T.A., Bellgowan, P.S., Springer, J.A., Kaufman, J.N. Possing, E.T., 2000. Human temporal lobe activation by speech and nonspeech sounds. Cereb. Cortex 10, 512-528.

Binder, J., Desai, R., Graves, W., Conant, L., 2009. Where is the semantic system? A critical review and meta-analysis of 120 functional neuroimaging studies. Cereb. Cortex 19 $2767-2796$

Bogen, J.E., Bogen, G.M., 1976. Wernicke's region, where is it? Ann. N. Y. Acad. Sci. 280, 834-843.

Broca, P., 1861. Remarques sur le siége de la faculté du langage articulé; suives d'une observation d'aphemie. Bull. Soc. Anat. Paris 6, 330-357.

Brown, S., Martinez, M.J., Parsons, L.M., 2006. Music and language side by side in the brain: a PET study of the generation of melodies and sentences. Eur. J. Neurosci. 23, 2791-2803.

Cai, Q., Paulignan, Y., Brysbaert, M., Ibarrola, D., Nazir, T.A., 2010. The left ventral occipitotemporal response to words depends on language lateralization but not on visua familiarity. Cereb. Cortex 20, 1153-1163.

Cardillo, E.R., Watson, C.E., Schmidt, G.L., Kranjec, A., Chatterjee, A., 2012. From novel to familiar: tuning the brain for metaphors. NeuroImage 59, 3212-3221.

Christensen, K.R., Wallentin, M., 2011. The locative alternation: distinguishing linguistic processing cost from error signals in Broca's region. Neurolmage 56, 1622-1631.

Christensen, K.R., Kizach, J., Nyvad, A.M., 2013. The processing of syntactic islands - an fMRI study. J. Neurolinguistics 26, 239-251.

Clark, A., 2013. Whatever next? Predictive brains, situated agents, and the future of cognitive science. Behav. Brain Sci. 36, 181-204.

Clements, A.M., Rimrodt, S.L., Abel, J.R., Blankner, J.G., Mostofsky, S.H., Pekar, J.J., Denckla, M.B., Cutting, L.E., 2006. Sex differences in cerebral laterality of language and visuospatial processing. Brain Lang. 98, 150-158.

Cohen, J.D., Perlstein, W.M., Braver, T.S., Nystrom, L.E., Noll, D.C., Jonides, J., Smith, E.E. 1997. Temporal dynamics of brain activation during a working memory task. Nature 386, 604-608.

Dehaene, S., Cohen, L., 2011. The unique role of the visual word form area in reading. Trends Cogn. Sci. 15, 254-262.

Dehaene, S., Naccache, L., Cohen, L., Bihan, D.L., Mangin, J.F., Poline, J.B., Rivière, D., 2001. Cerebral mechanisms of word masking and unconscious repetition priming. Nat. Neurosci. 4, 752-758.

Dehaene, S., Le Clec, H.G., Poline, J.-B., Le Bihan, D., Cohen, L., 2002. The visual word form area: a prelexical representation of visual words in the fusiform gyrus. Neuroreport 13, 321-325.

Dehaene, S., Nakamura, K., Jobert, A., Kuroki, C., Ogawa, S., Cohen, L., 2010a. Why do children make mirror errors in reading? Neural correlates of mirror invariance in the visual word form area. NeuroImage 49, 1837-1848.

Dehaene, S., Pegado, F., Braga, L.W., Ventura, P., Nunes Filho, G., Jobert, A., DehaeneLambertz, G., Kolinsky, R., Morais, J., Cohen, L., 2010b. How learning to read changes the cortical networks for vision and language. Science 330, 1359-1364.

Deppe, M., Knecht, S., Papke, K., Lohmann, H., Fleischer, H., Heindel, W., Ringelstein E.B., Henningsen, H., 2000. Assessment of hemispheric language lateralization: a comparison between fMRI and fTCD. J. Cereb. Blood Flow Metab. 20, 263-268.

Dronkers, N.F., Wilkins, D.P., Van Valin, R.D., Redfern, B.B., Jaeger, J.J., 2004. Lesion analysis of the brain areas involved in language comprehension. Cognition 92, 145-177.

Fedorenko, E., Duncan, J., Kanwisher, N., 2013. Broad domain generality in focal regions of frontal and parietal cortex. Proc. Natl. Acad. Sci. U. S. A. 110, 16616-16621.

Ferstl, E.C., Neumann, J., Bogler, C., Von Cramon, D.Y., 2008. The extended language network: a meta-analysis of neuroimaging studies on text comprehension. Hum. Brain Mapp. 29, 581-593.

Fiebach, C.J., Friederici, A.D., Müller, K., Von Cramon, D.Y., 2002. fMRI evidence for dua routes to the mental lexicon in visual word recognition. J. Cogn. Neurosci. 14, 11-23.

Fiebach, C.J., Schlesewsky, M., Lohmann, G., Von Cramon, D.Y., Friederici, A.D., 2005 Revisiting the role of Broca's area in sentence processing: syntactic integration versus syntactic working memory. Hum. Brain Mapp. 24, 79-91.

Friston, K., 2010. The free-energy principle: a unified brain theory? Nature reviews. Neuroscience 11, 127-138.

Gannon, P.J., Holloway, R.L., Broadfield, D.C., Braun, A.R., 1998. Asymmetry of chimpanzee planum temporale: humanlike pattern of Wernicke's brain language area homolog. Science 279, 220-222.

Geschwind, N., 1970. The organization of language and the brain. Science 170, 940-944.

Geschwind, N., Levitsky, W., 1968. Human brain: left-right asymmetries in tempora speech region. Science 161, 186-187.

Gonzalez-Castillo, J., Saad, Z.S., Handwerker, D.A., Inati, S.J., Brenowitz, N., Bandettini, P.A., 2012. Whole-brain, time-locked activation with simple tasks revealed using massive averaging and model-free analysis. Proc. Natl. Acad. Sci. U. S. A. 109 5487-5492.

Grodzinsky, Y., Santi, A., 2008. The battle for Broca's region. Trends Cogn. Sci. 12, 474-480.

Gusnard, D.A., Raichle, M.E., 2001. Searching for a baseline: functional imaging and the resting human brain. Nat. Rev. Neurosci. 2, 685-694.

January, D., Trueswell, J.C., Thompson-Schill, S.L., 2009. Co-localization of stroop and syntactic ambiguity resolution in Broca's area: implications for the neural basis of sentence processing. J. Cogn. Neurosci. 21, 2434-2444.

Jones, S.E., Mahmoud, S.Y., Phillips, M.D., 2011. A practical clinical method to quantify language lateralization in fMRI using whole-brain analysis. Neurolmage 54, 2937-2949.

Kahneman, D., Knetsch, J.L., Thaler, R.H., 1991. Anomalies: the endowment effect, loss aversion, and status quo bias. J. Econ. Perspect. 5, 193-206.

Kelly, A., Heathcote, A., Heath, R., Longstaff, M., 2001. Response-time dynamics: evidence for linear and low-dimensional nonlinear structure in human choice sequences. Q. J. Exp. Psychol. A 54, 805-840.

Knecht, S., Deppe, M., Dräger, B., Bobe, L., Lohmann, H., Ringelstein, E., Henningsen, H. 2000a. Language lateralization in healthy right-handers. Brain 123 (Pt 1), 74-81.
Knecht, S., Dräger, B., Deppe, M., Bobe, L., Lohmann, H., Flöel, A., Ringelstein, E.B., Henningsen, H., 2000b. Handedness and hemispheric language dominance in healthy humans. Brain 123 (Pt 12), 2512-2518.

Kristensen, L.B., Wallentin, M., 2014. Putting Broca's region into context - fMRI evidence for a role in predictive language processing. In: Willems, R. (Ed.), Towards a Cognitive Neuroscience of Natural Language Use. Cambridge University Press, Cambridge (in press).

Kristensen, L., Engberg-Pedersen, E., Nielsen, A., Wallentin, M., 2013. The influence of context on word order processing - an fMRI study. J. Neurolinguistics 26, 73-88.

Kristensen, L.B., Engberg-Pedersen, E., Wallentin, M., 2014. Context influences word order predictions in Broca's region. J. Cogn. Neurosci. (in press) http://dx.doi.org/10.1162/ jocn_a 00681.

Kruggel, F., von Cramon, D.Y., 1999a. Modeling the hemodynamic response in single-trial functional MRI experiments. Magn. Reson. Med. 42, 787-797.

Kruggel, F., von Cramon, D.Y., 1999b. Temporal properties of the hemodynamic response in functional MRI. Hum. Brain Mapp. 8, 259-271.

Laming, D., 1979. Autocorrelation of choice-reaction times. Acta Psychol. 43, 381-412.

Lehéricy, S., Cohen, L., Bazin, B., Samson, S., Giacomini, E., Rougetet, R., Hertz-Pannier, L., Le Bihan, D., Marsault, C., Baulac, M., 2000. Functional MR evaluation of temporal and frontal language dominance compared with the Wada test. Neurology 54, $1625-1633$

Levy, R., 2008. Expectation-based syntactic comprehension. Cognition 106, 1126-1177.

Logothetis, N.K., 2008. What we can do and what we cannot do with fMRI. Nature 453, 869-878.

McCandliss, B.D., Cohen, L., Dehaene, S., 2003. The visual word form area: expertise for reading in the fusiform gyrus. Trends Cogn. Sci. 7, 293-299.

Nicolle, A., Fleming, S.M., Bach, D.R., Driver, J., Dolan, R.J., 2011. A regret-induced status quo bias. J. Neurosci. 31, 3320-3327.

Novick, J.M., Trueswell, J.C., Thompson-Schill, S.L., 2010. Broca's area and language processing: evidence for the cognitive control connection. Linguist. Lang. Compass 4, 906-924.

Obleser, J., Kotz, S., 2010. Expectancy constraints in degraded speech modulate the language comprehension network. Cereb. Cortex 20,633.

Ojemann, G.A., 1991. Cortical organization of language. J. Neurosci. 11, 2281-2287.

Orfanidou, E., Marslen-Wilson, W.D., Davis, M.H., 2006. Neural response suppression predicts repetition priming of spoken words and pseudowords. J. Cogn. Neurosci. 18, $1237-1252$

Pedersen, P.M., Jørgensen, H.S., Nakayama, H., Raaschou, H.O., Olsen, T.S., 1995. Aphasia in acute stroke: incidence, determinants, and recovery. Ann. Neurol. 38, 659-666.

Pedersen, P.M.O.L., Vinter, K., Olsen, T.S.O.J., 2004. Aphasia after stroke: type, severity and prognosis. Cerebrovasc. Dis. 17, 35-43.

Penny, W., Holmes, A.P., 2007. Random effects analysis. In: Friston, K.J., Ashburner, J., Kiebel, S., Nichols, T., Penny, W. (Eds.), Statistical Parametric Mapping: The Analysis of Functional Brain Images. Academic Press, London, pp. 156-165.

Perry, L.K., Lupyan, G., 2014. The role of language in multi-dimensional categorization: evidence from transcranial direct current stimulation and exposure to verbal labels. Brain Lang. 135C, 66-72.

Petersen, B., Gjedde, A., Wallentin, M., Vuust, P., 2013. Cortical plasticity after cochlear implantation. Neural Plast. 2013, 1-11.

Price, C., 2000. The anatomy of language: contributions from functional neuroimaging. Journal of Anatomy, vol. 197, pp. 335-359.

Price, C.J., Devlin, J.T., 2003. The myth of the visual word form area. NeuroImage 19, 473-481.

Pujol, J., Deus, J., Losilla, J.M., Capdevila, A., 1999. Cerebral lateralization of language in normal left-handed people studied by functional MRI. Neurology 52, 1038-1043.

Raichle, M.E., Fiez, J.A., Videen, T.O., MacLeod, A.M., Pardo, J.V., Fox, P.T., Petersen, S.E., 1994. Practice-related changes in human brain functional anatomy during nonmotor learning. Cereb. Cortex 4, 8-26.

Rasmussen, T., Milner, B., 1977. The role of early left-brain injury in determining lateralization of cerebral speech functions. Ann. N. Y. Acad. Sci. 299, 355-369.

Schacter, D.L., Buckner, R.L., 1998. Priming and the brain. Neuron 20, 185-195.

Schnur, T.T., Schwartz, M.F., Kimberg, D.Y., Hirshorn, E., Coslett, H.B., Thompson-Schill, S.L., 2009. Localizing interference during naming: convergent neuroimaging and neuropsychological evidence for the function of Broca's area. Proc. Natl. Acad. Sci. U. S. A. 106, 322-327.

Seghier, M.L., 2008. Laterality index in functional MRI: methodological issues. Magn. Reson. Imaging 26, 594-601.

Snyder, H.R., Feigenson, K., Thompson-Schill, S.L., 2007. Prefrontal cortical response to conflict during semantic and phonological tasks. J. Cogn. Neurosci. 19, 761-775.

Spivey, M., 2007. The Continuity of Mind (Oxford Psychology). Oxford University Press, Oxford.

Springer, J.A., Binder, J.R., Hammeke, T.A., Swanson, S.J., Frost, J.A., Bellgowan, P.S., Brewer, C.C., Perry, H.M., Morris, G.L., Mueller, W.M., 1999. Language dominance in neurologically normal and epilepsy subjects: a functional MRI study. Brain 122 (Pt 11), 2033-2046.

Starrfelt, R., Gerlach, C., 2007. The visual what for area: words and pictures in the left fusiform gyrus. NeuroImage 35, 334-342.

Suarez, R.O., Whalen, S., Nelson, A.P., Tie, Y., Meadows, M.-E., Radmanesh, A., Golby, A.J., 2009. Threshold-independent functional MRI determination of language dominance: a validation study against clinical gold standards. Epilepsy Behav. 16, 288-297.

Sudre, G., Pomerleau, D., Palatucci, M., Wehbe, L., Fyshe, A., Salmelin, R., Mitchell, T., 2012. Tracking neural coding of perceptual and semantic features of concrete nouns. Neurolmage 62, 451-463.

Summerfeld, C., Wyart, V., Johnen, V.M., de Gardelle, V., 2011. Human scalp electroencephalography reveals that repetiti suppression varies with expectation. Front. Hum. Neurosci. 5, 67. 
Summerfield, C., Trittschuh, E.H., Monti, J.M., Mesulam, M.M., Egner, T., 2008. Neural repetition suppression reflects fulfilled perceptual expectations. Nat. Neurosci. 11, 1004-1006.

Szaflarski, J.P., Binder, J.R., Possing, E.T., McKiernan, K.A., Ward, B.D., Hammeke, T.A., 2002. Language lateralization in left-handed and ambidextrous people: fMRI data. Neurology 59, 238-244.

Szaflarski, J.P., Rajagopal, A., Altaye, M., Byars, A.W., Jacola, L., Schmithorst, V.J., Schapiro, M.B., Plante, E., Holland, S.K., 2012. Left-handedness and language lateralization in children. Brain Res. 1433, 85-97.

Szwed, M., Dehaene, S., Kleinschmidt, A., Eger, E., Valabrègue, R., Amadon, A., Cohen, L., 2011. Specialization for written words over objects in the visual cortex. NeuroImage 56, 330-344.

Tettamanti, M., Rotondi, I., Perani, D., Scotti, G., Fazio, F., Cappa, S.F., Moro, A., 2009. Syntax without language: neurobiological evidence for cross-domain syntactic computations. Cortex 45, 825-838.

Thompson-Schill, S.L., D'Esposito, M., Kan, I.P., 1999. Effects of repetition and competition on activity in left prefrontal cortex during word generation. Neuron 23, 513-522.

Todorovic, A., van Ede, F., Maris, E., de Lange, F.P., 2011. Prior expectation mediates neura adaptation to repeated sounds in the auditory cortex: an MEG study. J. Neurosci. 31, 9118-9123.

Turken, A.U., Dronkers, N.F., 2011. The neural architecture of the language comprehension network: converging evidence from lesion and connectivity analyses. Front. Syst. Neurosci. 5,1

Tzourio-Mazoyer, N., Landeau, B., Papathanassiou, D., Crivello, F., Etard, O., Delcroix, N., Mazoyer, B., Joliot, M., 2002. Automated anatomical labeling of activations in SPM using a macroscopic anatomical parcellation of the MNI MRI single-subject brain. Neurolmage 15, 273-289.

van de Meerendonk, N., Rueschemeyer, S.-A., Kolk, H.H.J., 2013. Language comprehension interrupted: both language errors and word degradation activate Broca's area. Brain Lang. 126, 291-301.

Van Orden, G.C., Holden, J.G., Turvey, M.T., 2003. Self-organization of cognitive performance. J. Exp. Psychol. Gen. 132, 331-350.

Vigneau, M., Beaucousin, V., Herve, P., Duffau, H., Crivello, F., Houde, O., Mazoyer, B. Tzourio-Mazoyer, N., 2006. Meta-analyzing left hemisphere language areas: phonology, semantics, and sentence processing. Neurolmage 30, 1414-1432.

Vigneau, M., Beaucousin, V., Hervé, P.-Y., Jobard, G., Petit, L., Crivello, F., Mellet, E., Zago, L. Mazoyer, B., Tzourio-Mazoyer, N., 2011. What is right-hemisphere contribution to phonological, lexico-semantic, and sentence processing? Insights from a metaanalysis. NeuroImage 54, 577-593.

Vinckier, F., Dehaene, S., Jobert, A., Dubus, J.P., Sigman, M., Cohen, L., 2007. Hierarchical coding of letter strings in the ventral stream: dissecting the inner organization of the visual word-form system. Neuron 55, 143-156.

Vuust, P., Roepstorff, A., 2008. Listen up! Polyrhythms in brain and music. Cogn. Semiot. 3, 131-159.

Vuust, P., Pallesen, K., Bailey, C., van Zuijen, T., Gjedde, A., Roepstorff, A., Østergaard, L., 2005. To musicians, the message is in the meter pre-attentive neuronal responses to incongruent rhythm are left-lateralized in musicians. NeuroImage 24, 560-564.

Vuust, P., Roepstorff, A., Wallentin, M., Mouridsen, K., Østergaard, L., 2006. It don't mean a thing... keeping the rhythm during polyrhythmic tension, activates language areas (BA 47). Neurolmage 31, 832-841.

Vuust, P., Wallentin, M., Mouridsen, K., Ostergaard, L., Roepstorff, A., 2011. Tapping polyrhythms in music activates language areas. Neurosci. Lett. 494, 211-216.

Wallentin, M., 2009. Putative sex differences in verbal abilities and language cortex: a critical review. Brain Lang. 108, 175-183.

Wallentin, M., Roepstorff, A., Glover, R., Burgess, N., 2006. Parallel memory systems for talking about location and age in precuneus, caudate and Broca's region. Neurolmage 32, 1850-1864.

Wallentin, M., Weed, E., Østergaard, L., Mouridsen, K., Roepstorff, A., 2008. Accessing the mental space - spatial working memory processes for language and vision overlap in precuneus. Hum. Brain Mapp. 29, 524-532.

Wallentin, M., Nielsen, A.H., Vuust, P., Dohn, A., Roepstorff, A., Lund, T.E., 2011. Amygdala and heart rate variability responses from listening to emotionally intense parts of a story. Neurolmage 58, 963-973.

Wernicke, C., 1874. Der aphasiche Symptomenkomplex. Cohen and Weigert, Breslau, Poland.

Wise, R.J., Scott, S.K., Blank, S.C., Mummery, C.J., Murphy, K., Warburton, E.A., 2001 Separate neural subsystems within "Wernicke's area". Brain 124, 83-95.

Woermann, F.G., Jokeit, H., Luerding, R., Freitag, H., Schulz, R., Guertler, S., Okujava, M. Wolf, P., Tuxhorn, I., Ebner, A., 2003. Language lateralization by Wada test and fMRI in 100 patients with epilepsy. Neurology 61, 699-701.

Worsley, K.J., Friston, K.J., 1995. Analysis of fMRI time-series revisited - again. NeuroImage 2, 173-181. 